\title{
Iterated Commutators of Multilinear Maximal Square Functions on Some Function Spaces
}

\author{
Zengyan Si*, Qingying Xue and Pu Zhang
}

\begin{abstract}
In this paper, the iterated commutators of multilinear maximal square function and pointwise multiplication with functions in Lipschitz spaces are studied. Some new estimates for the iterated commutators with kernels satisfying some Dini type conditions on Lebesgue spaces, homogenous Lipschitz spaces and homogenous Triebel-Lizorkin spaces will be given, respectively.
\end{abstract}

\section{Introduction}

Commutators of singular integral operators with Lipschitz functions have been the subject of many recent articles. In 1995, Paluszyński [16] proved that the commutators generated by Calderón-Zygmund operators and Lipschitz functions are bounded from Lebesgue space to Lebesgue space and to homogenous Triebel-Lizorkin space. For the commutators in the case of the multilinear Calderón-Zygmund operators with the kernel of standard estimates, Wang and Xu 24] and Mo and Lu [15] got the boundedness on Lebesgue space, homogenous Triebel-Lizorkin space and Lipschitz spaces respectively.

Recently, in the theory of multilinear operators, efforts have been made to remove or replace the smoothness condition assumed on the kernels. Among these achievements, we mention the nice works of Bui and Duong [1], Grafakos, Liu and Yang [9], Maldonado and Naibo [14], Lu and Zhang [13], Tomita [23], Grafakos, Si [10] and more recent work of Grafakos, He and Honzík [8]. In 2017, Sun and Zhang [22 got the boundedness for commutators of multilinear Calderón-Zygmund operators with kernels of Dini type from product of Lebesgue spaces into Lebesgue spaces, Lipschitz spaces and homogenous Triebel-Lizorkin spaces, which extend some previous results. Very recently, the study for commutators of maximal operator of multilinear singular integral with kernels of Dini type was given by Si and Zhang 21.

It was well-known that the Calderón-Zygmund operators and the Littlewood-Paley operators have very close relationship. The multilinear square functions were introduced

Received August 28, 2019; Accepted January 7, 2020.

Communicated by Duy-Minh Nhieu.

2010 Mathematics Subject Classification. 42B25, 47G10.

Key words and phrases. multilinear maximal square functions, multilinear square functions, iterated commutators.

${ }^{*}$ Corresponding author. 
and first studied by Coifman and Meyer [4] (studied the $L^{2}$ estimate by using the notion of Carleson measures), and later by Yabuta [27] (obtained the $L^{p}(p \geq 1)$ boundedness and BMO type estimates by weaking the assumptions in [4]). In 2001, Sato and Yabuta 17 studied the $\left(L^{p_{1}} \times \cdots \times L^{p_{m}}, L^{p}\right)$ boundedness with $p \geq 1 / m$ for $m \geq 2$. The study of this subject was recently enjoyed a resurgence of renewed interest and activity. In 2016, Si and Xue [19 studied the bounded properties of multilinear square function and multilinear maximal square function with kernels satisfying Dini type conditions on Lebesgue and Morrey type spaces respectively. In 2018, Si and Xue [20] got the boundedness for iterated commutators of multilinear square functions with Dini-type kernels from product of Lebesgue spaces into Lebesgue spaces, Lipschitz spaces and homogenous Triebel-Lizorkin spaces, which can be seen as the vector-valued extension of the previous results in [22]. For other recent works about multilinear Littlewood-Paley type operators, see [2, 18,25,26] and the references therein. For the applications of the theory of multilinear square functions in PDE and other fields, see $3,5,7,11,17,26,27$ and the references therein. In this paper we study the boundedness properties of the commutators of multilinear maximal square functions with kernels satisfying Dini type conditions. In order to state our results, we first prepare some notions and definitions.

For any $t \in(0, \infty)$, a locally integrable function $K_{t}(x, \vec{y})$ defined away from the diagonal $x=y_{1}=\cdots=y_{m}$ in $\left(\mathbb{R}^{n}\right)^{m+1}$ is called a kernel of type $\omega(t)$, if there is a positive constant $A$, such that the following conditions hold.

1. Size condition:

$$
\left(\int_{0}^{\infty}\left|K_{t}(x, \vec{y})\right|^{2} \frac{d t}{t}\right)^{1 / 2} \leq \frac{A}{\left(\sum_{j=1}^{m}\left|x-y_{j}\right|\right)^{m n}}
$$

2. Smoothness condition: Whenever $|z-x| \leq \frac{1}{m+1} \max _{1 \leq j \leq m}\left|x-y_{j}\right|$, it holds that

$$
\left(\int_{0}^{\infty}\left|K_{t}(z, \vec{y})-K_{t}(x, \vec{y})\right|^{2} \frac{d t}{t}\right)^{1 / 2} \leq \frac{A}{\left(\sum_{j=1}^{m}\left|x-y_{j}\right|\right)^{m n}} \omega\left(\frac{|z-x|}{\sum_{j=1}^{m}\left|x-y_{j}\right|}\right) .
$$

Whenever $\left|y_{j}-y_{j}^{\prime}\right| \leq \frac{1}{m+1} \max _{1 \leq j \leq m}\left|x-y_{j}\right|$, it holds that

$$
\begin{aligned}
& \left(\int_{0}^{\infty}\left|K_{t}(x, \vec{y})-K_{t}\left(x, y_{1}, \ldots, y_{j}^{\prime}, \ldots, y_{m}\right)\right|^{2} \frac{d t}{t}\right)^{1 / 2} \\
\leq & \frac{A}{\left(\sum_{j=1}^{m}\left|x-y_{j}\right|\right)^{m n}} \omega\left(\frac{\left|y_{j}-y_{j}^{\prime}\right|}{\sum_{j=1}^{m}\left|x-y_{j}\right|}\right) .
\end{aligned}
$$

The multilinear maximal square function of type $\omega(t)$ is defined by

$$
T^{*}(\vec{f})(x)=\sup _{\delta>0} T_{\delta}(\vec{f})(x)
$$


where $T_{\delta}$ is the truncated operator associated to $K_{t}$ defined by

$$
T_{\delta}(\vec{f})(x)=\left(\int_{0}^{\infty}\left|\int_{\sum_{i=1}^{m}\left|x-y_{i}\right|^{2}>\delta^{2}} K_{t}\left(x, y_{1}, \ldots, y_{m}\right) \prod_{j=1}^{m} f_{j}\left(y_{j}\right) d y_{1} \cdots d y_{m}\right|^{2} \frac{d t}{t}\right)^{1 / 2} .
$$

Let $\vec{b}=\left(b_{1}, \ldots, b_{m}\right)$ be a collection of locally integrable functions. We study the iterated commutator associated with $T^{*}$

$$
\begin{gathered}
T_{\Pi \vec{b}}^{*}(\vec{f})(x)=\sup _{\delta>0}\left(\int_{0}^{\infty} \mid \int_{\sum_{i=1}^{m}\left|x-y_{i}\right|^{2}>\delta^{2}} \prod_{j=1}^{m}\left[b_{j}(x)-b_{j}\left(y_{j}\right)\right] K_{t}\left(x, y_{1}, \ldots, y_{m}\right)\right. \\
\left.\times\left.\prod_{j=1}^{m} f_{j}\left(y_{j}\right) d y_{1} \cdots d y_{m}\right|^{2} \frac{d t}{t}\right)^{1 / 2} .
\end{gathered}
$$

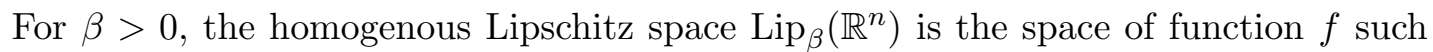
that

$$
\|f\|_{\operatorname{Lip}_{\beta}\left(\mathbb{R}^{n}\right)}=\sup _{x, h \in \mathbb{R}^{n}, h \neq 0} \frac{\left|\Delta_{h}^{[\beta]+1} f(x)\right|}{|h|^{\beta}}<\infty,
$$

where $\Delta_{h}^{k}$ denotes the $k$-th difference operator. The following characterizations for homogenous Lipschitz spaces and homogenous Triebel-Lizorkin spaces can be found in [16].

(i) For $0<\beta<1,1 \leq q<\infty$, we have

$$
\|f\|_{\operatorname{Lip}_{\beta}} \approx \sup _{Q} \frac{1}{|Q|^{1+n / \beta}} \int_{Q}\left|f-f_{Q}\right| \approx \sup _{Q} \frac{1}{|Q|^{n / \beta}}\left(\int_{Q}\left|f-f_{Q}\right|^{q}\right)^{1 / q} .
$$

(ii) For $0<\beta<1,1 \leq p<\infty$, we have

$$
\|f\|_{\dot{F}_{p}^{\beta, \infty}} \approx\left\|\sup _{Q} \frac{1}{|Q|^{1+n / \beta}} \int_{Q}\left|f-f_{Q}\right|\right\|_{L^{p}} .
$$

We always assume that $\omega(t):[0, \infty) \mapsto[0, \infty)$ is a nondecreasing function with $0<$ $\omega(1)<\infty$. For $a>0$, we say that $\omega \in \operatorname{Dini}(a)$ if

$$
|\omega|_{\operatorname{Dini}(a)}=\int_{0}^{1} \omega^{a}(t) \frac{d t}{t}<\infty
$$

The main results of this paper are as follows.

Theorem 1.1. Let $1 / q=1 / p_{1}+\cdots+1 / p_{m}-\beta / n$ and $\beta=\beta_{1}+\cdots+\beta_{m}$. Suppose that $0<q<\infty, 1<p_{1}, \ldots, p_{m}<\infty$ and $1 / p_{j}>\beta_{j} / n$. If $\omega \in \operatorname{Dini}(1)$ and $b_{j} \in \operatorname{Lip}_{\beta_{j}}$ with $0<\beta_{j}<1$ for $j=1, \ldots, m$, then

$$
\left\|T_{\Pi \vec{b}}^{*} \vec{f}\right\|_{L^{q}\left(\mathbb{R}^{n}\right)} \lesssim \prod_{i=1}^{m}\left\|b_{i}\right\|_{\operatorname{Lip}_{\beta_{i}}\left(\mathbb{R}^{n}\right)} \prod_{i=1}^{m}\left\|f_{i}\right\|_{L^{p_{i}\left(\mathbb{R}^{n}\right)}}
$$


Theorem 1.2. Let $1 / p=1 / p_{1}+\cdots+1 / p_{m}$ and $\beta=\beta_{1}+\cdots+\beta_{m}$. Suppose that $1<p_{1}, \ldots, p_{m}<\infty, 0<1 / p_{j}<\beta_{j} / n$ and $0<\beta-n / p<1$. If $b_{j} \in \operatorname{Lip}_{\beta_{j}}$ with $0<\beta_{j}<1$ for $j=1, \ldots, m$ and $\omega$ satisfies

$$
\int_{0}^{1} \frac{\omega(t)}{t^{1+\beta-n / p}} d t<\infty
$$

Then it holds that

$$
\left\|T_{\Pi \vec{b}}^{*} \vec{f}\right\|_{\operatorname{Lip}_{\beta-n / p}\left(\mathbb{R}^{n}\right)} \lesssim \prod_{i=1}^{m}\left\|b_{i}\right\|_{\operatorname{Lip}_{\beta_{i}}\left(\mathbb{R}^{n}\right)} \prod_{i=1}^{m}\left\|f_{i}\right\|_{L^{p_{i}\left(\mathbb{R}^{n}\right)}}
$$

Theorem 1.3. Let $1 / p=1 / p_{1}+\cdots+1 / p_{m}$ and $\beta=\beta_{1}+\cdots+\beta_{m}$. Suppose that $1<p_{1}, \ldots, p_{m}<\infty$. If $b_{j} \in \operatorname{Lip}_{\beta_{j}}$ with $0<\beta_{j}<1$ for $j=1, \ldots, m$ and $\omega$ satisfies

$$
\int_{0}^{1} \frac{\omega(t)}{t^{1+\beta}} d t<\infty
$$

Then it holds that

$$
\left\|T_{\Pi \vec{b}}^{*} \vec{f}\right\|_{\dot{F}_{p}^{\beta, \infty}\left(\mathbb{R}^{n}\right)} \lesssim \prod_{i=1}^{m}\left\|b_{i}\right\|_{\operatorname{Lip}_{\beta_{i}}\left(\mathbb{R}^{n}\right)} \prod_{i=1}^{m}\left\|f_{i}\right\|_{L^{p_{i}\left(\mathbb{R}^{n}\right)}} .
$$

Remark 1.4. If we let

$$
K_{t}\left(x, y_{1}, \ldots, y_{m}\right)= \begin{cases}K\left(x, y_{1}, \ldots, y_{m}\right) & \text { if } 1 \leq t \leq e \\ 0 & \text { otherwise }\end{cases}
$$

then $T_{\Pi \vec{b}}^{*}$ become the iterated commutators of maximal operator of multilinear singular integral with kernels of Dini type

$$
T_{\Pi \vec{b}}^{*}(\vec{f})(x)=\sup _{\delta>0}\left|\int_{\sum_{i=1}^{m}\left|x-y_{i}\right|^{2}>\delta^{2}} \prod_{j=1}^{m}\left[b_{j}(x)-b_{j}\left(y_{j}\right)\right] K\left(x, y_{1}, \ldots, y_{m}\right) \prod_{j=1}^{m} f_{j}\left(y_{j}\right) d \vec{y}\right|,
$$

where $d \vec{y}=d y_{1} \cdots d y_{m}$.

Throughout this paper, the notation $A \lesssim B$ stands for $A \leq C B$ for some positive constant $C$ independent of $A$ and $B$.

\section{Proof of Theorem 1.1}

Let $f$ be a locally integrable function. The fractional maximal function is define by

$$
M_{r, \beta} f(x)=\sup _{x \in Q}\left(\frac{1}{|Q|^{1-\beta r / n}} \int_{Q}|f(y)|^{r} d y\right)^{1 / r},
$$


when $r \geq 1$ and $0 \leq \beta<n / r$. If $\beta=0$ and $r=1$, then $M_{1,0} f=M f$ denotes the usual Hardy-Littlewood maximal function. For $\delta>0$, we denote $M_{\delta}$ by $M_{\delta} f=M\left(|f|^{\delta}\right)^{1 / \delta}$.

The sharp maximal function $M^{\sharp}$ is given by

$$
M^{\sharp} f(x)=\sup _{Q \ni x} \inf _{c} \frac{1}{|Q|} \int_{Q}|f(y)-c| d y \approx \sup _{Q \ni x} \frac{1}{|Q|} \int_{Q}\left|f(y)-f_{Q}\right| d y,
$$

where $f_{Q}$ is the average of $f$ over cube $Q$ and we denote $M_{\delta}^{\sharp}$ by $M_{\delta}^{\sharp} f(x)=M^{\sharp}\left(|f|^{\delta}\right)^{1 / \delta}(x)$.

Let $u, v \in C^{\infty}([0, \infty))$ such that $\left|u^{\prime}(t)\right| \lesssim t^{-1},\left|v^{\prime}(t)\right| \lesssim t^{-1}$ and satisfy

$$
\chi_{[2, \infty)}(t) \leq u(t) \leq \chi_{[1, \infty)}(t), \quad \chi_{[1,2]}(t) \leq v(t) \leq \chi_{[1 / 2,3]}(t)
$$

For simplicity, we denote

$$
\begin{aligned}
& K_{t, u, \eta}\left(x, y_{1}, \ldots, y_{m}\right)=K_{t}\left(x, y_{1}, \ldots, y_{m}\right) u\left(\frac{\left|x-y_{1}\right|+\cdots+\left|x-y_{m}\right|}{\eta}\right), \\
& K_{t, v, \eta}\left(x, y_{1}, \ldots, y_{m}\right)=K_{t}\left(x, y_{1}, \ldots, y_{m}\right) v\left(\frac{\left|x-y_{1}\right|+\cdots+\left|x-y_{m}\right|}{\eta}\right) .
\end{aligned}
$$

It is easy to see that $K_{t, u, \eta}$ and $K_{t, v, \eta}$ satisfy the size condition (1.1). Next, we show that the functions $K_{t, u, \eta}$ and $K_{t, v, \eta}$ satisfy some smooth properties.

Lemma 2.1. For any $j=0,1,2, \ldots, m$, we get

$$
\begin{aligned}
& \left(\int_{0}^{\infty}\left|K_{t, u, \eta}\left(y_{0}, \ldots, y_{j}, \ldots, y_{m}\right)-K_{t, u, \eta}\left(y_{0}, \ldots, y_{j}^{\prime}, \ldots, y_{m}\right)\right|^{2} \frac{d t}{t}\right)^{1 / 2} \\
\lesssim & \frac{\omega\left(\frac{\left|y_{j}-y_{j}^{\prime}\right|}{\left|y_{0}-y_{1}\right|+\cdots+\left|y_{0}-y_{m}\right|}\right)}{\left(\left|y_{0}-y_{1}\right|+\cdots+\left|y_{0}-y_{m}\right|\right)^{m n}}+\frac{\left|y_{j}-y_{j}^{\prime}\right|}{\left(\left|y_{0}-y_{1}\right|+\cdots+\left|y_{0}-y_{m}\right|\right)^{m n+1}}
\end{aligned}
$$

and

$$
\begin{aligned}
& \left(\int_{0}^{\infty}\left|K_{t, v, \eta}\left(y_{0}, \ldots, y_{j}, \ldots, y_{m}\right)-K_{t, v, \eta}\left(y_{0}, \ldots, y_{j}^{\prime}, \ldots, y_{m}\right)\right|^{2} \frac{d t}{t}\right)^{1 / 2} \\
\lesssim & \frac{\omega\left(\frac{\left|y_{j}-y_{j}^{\prime}\right|}{\left|y_{0}-y_{1}\right|+\cdots+\left|y_{0}-y_{m}\right|}\right)}{\left(\left|y_{0}-y_{1}\right|+\cdots+\left|y_{0}-y_{m}\right|\right)^{m n}}+\frac{\left|y_{j}-y_{j}^{\prime}\right|}{\left(\left|y_{0}-y_{1}\right|+\cdots+\left|y_{0}-y_{m}\right|\right)^{m n+1}},
\end{aligned}
$$

whenever $\left|y_{j}-y_{j}^{\prime}\right| \leq \frac{1}{m+1} \max _{0 \leq j \leq m}\left|y_{0}-y_{j}\right|$.

Proof. We just give the estimate for $K_{t, u, \eta}$, since $K_{t, v, \eta}$ can be estimated in a similar way with a little modifications. Without loss of generality, we assume $j=0$, then

$$
\begin{aligned}
& \left(\int_{0}^{\infty}\left|K_{t, u, \eta}\left(y_{0}, \ldots, y_{j}, \ldots, y_{m}\right)-K_{t, u, \eta}\left(y_{0}, \ldots, y_{j}^{\prime}, \ldots, y_{m}\right)\right|^{2} \frac{d t}{t}\right)^{1 / 2} \\
= & \left(\int_{0}^{\infty} \mid K_{t}\left(y_{0}, \vec{y}\right) u\left(\frac{\left|y_{0}-y_{1}\right|+\cdots+\left|y_{0}-y_{m}\right|}{\eta}\right)\right.
\end{aligned}
$$




$$
\begin{aligned}
& \left.-\left.K_{t}\left(y_{0}^{\prime}, \vec{y}\right) u\left(\frac{\left|y_{0}^{\prime}-y_{1}\right|+\cdots+\left|y_{0}^{\prime}-y_{m}\right|}{\eta}\right)\right|^{2} \frac{d t}{t}\right)^{1 / 2} \\
= & \left(\int_{0}^{\infty} \mid\left[K_{t}\left(y_{0}, \vec{y}\right)-K_{t}\left(y_{0}^{\prime}, \vec{y}\right)\right] u\left(\frac{\left|y_{0}^{\prime}-y_{1}\right|+\cdots+\left|y_{0}^{\prime}-y_{m}\right|}{\eta}\right)\right. \\
& \left.\quad-\left.K_{t}\left(y_{0}, \vec{y}\right)\left[u\left(\frac{\left|y_{0}^{\prime}-y_{1}\right|+\cdots+\left|y_{0}^{\prime}-y_{m}\right|}{\eta}\right)-u\left(\frac{\left|y_{0}-y_{1}\right|+\cdots+\left|y_{0}-y_{m}\right|}{\eta}\right)\right]\right|^{2} \frac{d t}{t}\right)^{1 / 2} \\
\lesssim & \left(\int_{0}^{\infty}\left|K_{t}\left(y_{0}, \vec{y}\right)-K_{t}\left(y_{0}^{\prime}, \vec{y}\right)\right|^{2} \frac{d t}{t}\right)^{1 / 2} \\
+ & \left(\int_{0}^{\infty} \mid K_{t}\left(y_{0}, y_{1}, \ldots, y_{m}\right)\right. \\
& \left.\times\left.\left[u\left(\frac{\left|y_{0}^{\prime}-y_{1}\right|+\cdots+\left|y_{0}^{\prime}-y_{m}\right|}{\eta}\right)-u\left(\frac{\left|y_{0}-y_{1}\right|+\cdots+\left|y_{0}-y_{m}\right|}{\eta}\right)\right]\right|^{2} \frac{d t}{t}\right)^{1 / 2} \\
\doteq & I I .
\end{aligned}
$$

By the fact $\left|y_{0}-y_{0}^{\prime}\right| \leq \frac{1}{m+1} \max _{0 \leq j \leq m}\left|y_{0}-y_{j}\right|$ and by the smoothness condition 1.2, we get

$$
I \lesssim \frac{1}{\left(\left|y_{0}-y_{1}\right|+\cdots+\left|y_{0}-y_{m}\right|\right)^{m n}} \omega\left(\frac{\left|y_{0}-y_{0}^{\prime}\right|}{\left|y_{0}-y_{1}\right|+\cdots+\left|y_{0}-y_{m}\right|}\right)
$$

Next, we shall use the following estimate (see Lemma 3.1 in 21]):

$$
\begin{aligned}
& \left|u\left(\frac{\left|y_{0}^{\prime}-y_{1}\right|+\cdots+\left|y_{0}^{\prime}-y_{m}\right|}{\eta}\right)-u\left(\frac{\left|y_{0}-y_{1}\right|+\cdots+\left|y_{0}-y_{m}\right|}{\eta}\right)\right| \\
\lesssim & \frac{\left|y_{0}-y_{0}^{\prime}\right|}{\left|y_{0}-y_{1}\right|+\cdots+\left|y_{0}-y_{m}\right|} .
\end{aligned}
$$

This together with the size condition 1.1 implies that

$$
I I \lesssim \frac{\left|y_{0}-y_{0}^{\prime}\right|}{\left(\left|y_{0}-y_{1}\right|+\cdots+\left|y_{0}-y_{m}\right|\right)^{m n+1}} .
$$

This finishes the proof of Lemma 2.1

Associate with $K_{u, \eta}$ and $K_{v, \eta}$, we define two maximal operators $U^{*}(\vec{f})(x)=\sup _{\eta>0}$ $\left|U_{\eta}(\vec{f})(x)\right|$ and $V^{*}(\vec{f})(x)=\sup _{\eta>0}\left|V_{\eta}(\vec{f})(x)\right|$, where

$$
\begin{aligned}
& U_{\eta}(\vec{f})(x)=\left(\int_{0}^{\infty}\left|\int_{\left(\mathbb{R}^{n}\right)^{m}} K_{t, u, \eta}(x, \vec{y}) \prod_{i=1}^{m} f_{i}\left(y_{i}\right) d \vec{y}\right|^{2} \frac{d t}{t}\right)^{1 / 2}, \\
& V_{\eta}(\vec{f})(x)=\left(\int_{0}^{\infty}\left|\int_{\left(\mathbb{R}^{n}\right)^{m}} K_{t, v, \eta}(x, \vec{y}) \prod_{i=1}^{m} f_{i}\left(y_{i}\right) d \vec{y}\right|^{2} \frac{d t}{t}\right)^{1 / 2} .
\end{aligned}
$$

As a consequence of Lemma 2.1, the boundedness of multilinear maximal square functions with kernels of Dini type (see Theorem 6 in [19]), and the estimates of multilinear maximal square functions with non-smooth kernels (see Theorem 4.1 in [12]), we have 
Lemma 2.2. Let $1 / p=1 / p_{1}+\cdots+1 / p_{m}$. We have

(1) If $1<p_{1}, \ldots, p_{m}<\infty$, then

$$
\left\|U^{*} \vec{f}\right\|_{L^{p}\left(\mathbb{R}^{n}\right)} \lesssim \prod_{i=1}^{m}\left\|f_{i}\right\|_{L^{p_{i}\left(\mathbb{R}^{n}\right)} .}
$$

(2) If $1 \leq p_{1}, \ldots, p_{m}<\infty$, then

$$
\left\|U^{*} \vec{f}\right\|_{L^{p, \infty}\left(\mathbb{R}^{n}\right)} \lesssim \prod_{i=1}^{m}\left\|f_{i}\right\|_{L^{p_{i}\left(\mathbb{R}^{n}\right)}} .
$$

Similar estimates hold for $V^{*}$.

Given a collection of locally integrable functions $\vec{b}=\left(b_{1}, \ldots, b_{m}\right)$, we define the commutator $T_{\Sigma \vec{b}}^{*}$ by

$$
T_{\Sigma \vec{b}}^{*}\left(f_{1}, \ldots, f_{m}\right)=\sum_{j=1}^{m} T_{\vec{b}}^{* j}(\vec{f}),
$$

where $\vec{f}=\left(f_{1}, \ldots, f_{m}\right)$ and $T_{\vec{b}}^{* j}$ is the commutator of $b_{j}$ and $T^{*}$ in the $j$-th entry of $T^{*}$, that is,

$$
T_{\vec{b}}^{* j}(\vec{f})(x)=\left[b_{j}, T^{*}\right]_{j}(\vec{f})(x)=\sup _{\delta>0}\left|b_{j}(x) T_{\delta}\left(f_{1}, \ldots, f_{m}\right)(x)-T_{\delta}\left(f_{1}, \ldots, b_{j} f_{j}, \ldots, f_{m}\right)(x)\right| .
$$

The key role in the proof of the main results is played by the maximal operators $U_{\Pi b}^{*}$ and $V_{\Pi b}^{*}$, which are given by

$$
\begin{aligned}
& U_{\Pi \vec{b}}^{*}(\vec{f})(x)=\sup _{\eta>0}\left(\int_{0}^{\infty}\left|\int_{\left(\mathbb{R}^{n}\right)^{2}} K_{t, u, \eta}\left(x, y_{1}, y_{2}\right) \prod_{j=1}^{2}\left[b_{j}(x)-b_{j}\left(y_{j}\right)\right] \prod_{i=1}^{2} f_{i}\left(y_{i}\right) d \vec{y}\right|^{2} \frac{d t}{t}\right)^{1 / 2}, \\
& V_{\Pi \vec{b}}^{*}(\vec{f})(x)=\sup _{\eta>0}\left(\int_{0}^{\infty}\left|\int_{\left(\mathbb{R}^{n}\right)^{2}} K_{t, v, \eta}\left(x, y_{1}, y_{2}\right) \prod_{j=1}^{2}\left[b_{j}(x)-b_{j}\left(y_{j}\right)\right] \prod_{i=1}^{2} f_{i}\left(y_{i}\right) d \vec{y}\right|^{2} \frac{d t}{t}\right)^{1 / 2} .
\end{aligned}
$$

It is easy to see that $T_{\Pi b}^{*}(\vec{f}) \leq U_{\Pi b}^{*}(\vec{f})(x)+V_{\Pi b}^{*}(\vec{f})(x)$ and $T^{*}(\vec{f}) \leq U^{*}(\vec{f})(x)+V^{*}(\vec{f})(x)$. To prove Theorem 1.1, we need the following estimates for $T_{\Pi \vec{b}}^{*}$. We just consider the case $m=2$ for simplicity, our method still hold for general $m$ with a little bit of modifications.

Lemma 2.3. Let $T^{*}$ be a multilinear maximal square function of type $\omega(t)$ with $\omega \in$ Dini(1). Then, we have

(i) Let $b_{1} \in \operatorname{Lip}_{\beta_{1}}$ and $b_{2} \in \operatorname{Lip}_{\beta_{2}}$ with $0<\beta_{1}, \beta_{2}<1,0<\delta<\epsilon<1 / 2$, then

$$
\begin{aligned}
& M_{\delta}^{\sharp} T_{\Pi \vec{b}}^{*}\left(f_{1}, f_{2}\right)(x) \\
\lesssim & \prod_{i=1}^{2}\left\|b_{i}\right\|_{\operatorname{Lip}_{\beta_{i}}} M_{\epsilon, \beta}\left(T^{*}\left(f_{1}, f_{2}\right)\right)(x)+\left\|b_{1}\right\|_{\operatorname{Lip}_{\beta_{1}}} M_{\epsilon, \beta_{1}}\left(T_{\vec{b}}^{* 2}\left(f_{1}, f_{2}\right)\right)(x) \\
& +\left\|b_{2}\right\|_{\operatorname{Lip}_{\beta_{1}}} M_{\epsilon, \beta_{2}}\left(T_{\vec{b}}^{* 1}\left(f_{1}, f_{2}\right)\right)(x)+\prod_{i=1}^{2}\left\|b_{i}\right\|_{\operatorname{Lip}_{\beta_{i}}} M_{1, \beta_{1}}\left(f_{1}\right)(x) M_{1, \beta_{2}}\left(f_{2}\right)(x) .
\end{aligned}
$$


(ii) Suppose that $b_{j} \in \operatorname{Lip}_{\beta}, j=1,2,0<\beta<1$ and $0<\delta<\epsilon<1 / 2<1<n / \beta$, then

$$
\begin{aligned}
& M_{\delta}^{\sharp} T_{\Sigma \vec{b}}^{*}\left(f_{1}, f_{2}\right)(x) \\
\lesssim & \|b\|_{\operatorname{Lip}_{\beta}}\left\{M_{\epsilon, \beta}\left(T^{*}\left(f_{1}, f_{2}\right)\right)(x)+M_{1, \beta}\left(f_{1}\right)(x) M\left(f_{2}\right)(x)+M_{1, \beta}\left(f_{2}\right)(x) M\left(f_{1}\right)(x)\right\} .
\end{aligned}
$$

Proof. We should point out that the proof of this lemma is similar to that of Lemma 3.3 in [21], so we just give a brief step and the part of the proof that is different.

(i) We prove (2.1) holds for $U_{\Pi \vec{b}}^{*}$. Fix $x \in \mathbb{R}^{n}$, denote $Q=Q\left(x_{Q}, l\right)$ the cube centered at $x_{Q}$ and containing $x$ with side-length $l$. Denote $c=\sup _{\eta>0}\left|c_{\eta}\right|$ and $\left(b_{i}\right)_{Q^{*}}=$ $\frac{1}{\left|Q^{*}\right|} \int_{Q^{*}} b_{i}(y) d y$, where $Q^{*}=8 \sqrt{n} Q$. Then, we have

$$
\begin{aligned}
& \left(\left.\frac{1}{|Q|} \int_{Q}|| U_{\Pi \vec{b}}^{*}\left(f_{1}, f_{2}\right)(z)\right|^{\delta}-|c|^{\delta} \mid d z\right)^{1 / \delta} \\
\lesssim & \left(\left.\frac{1}{|Q|} \int_{Q}\left|U_{\Pi \vec{b}}^{*}\left(f_{1}, f_{2}\right)(z)-\sup _{\eta>0}\right| c_{\eta}\right|^{\delta} d z\right)^{1 / \delta} \\
\lesssim & \left(\frac{1}{|Q|} \int_{Q}\left|\left(b_{1}(z)-\left(b_{1}\right)_{Q^{*}}\right)\left(b_{2}(z)-\left(b_{2}\right)_{Q^{*}}\right) U^{*}\left(f_{1}, f_{2}\right)(z)\right|^{\delta} d z\right)^{1 / \delta} \\
& +\left(\frac{1}{|Q|} \int_{Q}\left|\left(b_{1}(z)-\left(b_{1}\right)_{Q^{*}}\right)\left[b_{2}, U^{*}\right]_{2}\left(f_{1}, f_{2}\right)(z)\right|^{\delta} d z\right)^{1 / \delta} \\
& +\left(\frac{1}{|Q|} \int_{Q}\left|\left(b_{2}(z)-\left(b_{2}\right)_{Q^{*}}\right)\left[b_{1}, U^{*}\right]_{1}\left(f_{1}, f_{2}\right)(z)\right|^{\delta} d z\right)^{1 / \delta} \\
& +\left(\frac{1}{|Q|} \int_{Q} \sup _{\eta>0}\left|U_{\eta}\left(\left(b_{1}-\left(b_{1}\right)_{Q^{*}}\right) f_{1},\left(b_{2}-\left(b_{2}\right)_{Q^{*}}\right) f_{2}\right)(z)-c_{\eta}\right|^{\delta} d z\right)^{1 / \delta} \\
\doteq & \mathfrak{T}_{1}+\mathfrak{T}_{2}+\mathfrak{T}_{3}+\mathfrak{T}_{4} .
\end{aligned}
$$

By Hölder's inequality, we get

$$
\mathfrak{T}_{1} \lesssim \prod_{i=1}^{2}\left\|b_{i}\right\|_{\operatorname{Lip}_{\beta_{i}}} M_{\epsilon, \beta}\left(U^{*}\left(f_{1}, f_{2}\right)\right)(x)
$$

and

$$
\mathfrak{T}_{2}+\mathfrak{T}_{3} \lesssim\left\|b_{1}\right\|_{\operatorname{Lip}_{\beta_{1}}} M_{\epsilon, \beta_{1}}\left(\left[b_{2}, U^{*}\right]_{2}\left(f_{1}, f_{2}\right)\right)(x)+\left\|b_{2}\right\|_{\operatorname{Lip}_{\beta_{2}}} M_{\epsilon, \beta_{2}}\left(\left[b_{1}, U^{*}\right]_{1}\left(f_{1}, f_{2}\right)\right)(x)
$$

It remains to estimate the last term $\mathfrak{T}_{4}$. Take now

$$
c_{\eta}=U_{\eta}\left(\left(b_{1}-\left(b_{1}\right)_{Q^{*}}\right) f_{1}^{\infty},\left(b_{2}-\left(b_{2}\right)_{Q^{*}}\right) f_{2}^{\infty}\right)(x) .
$$

Then $\mathfrak{T}_{4} \leq \mathfrak{T}_{41}+\mathfrak{T}_{42}+\mathfrak{T}_{43}+\mathfrak{T}_{44}$, where

$$
\mathfrak{T}_{41}=\left(\frac{1}{|Q|} \int_{Q}\left|U^{*}\left(\left(b_{1}-\left(b_{1}\right)_{Q^{*}}\right) f_{1}^{0},\left(b_{2}-\left(b_{2}\right)_{Q^{*}}\right) f_{2}^{0}\right)(z)\right|^{\delta} d x\right)^{1 / \delta},
$$




$$
\begin{aligned}
\mathfrak{T}_{42}= & \left(\frac{1}{|Q|} \int_{Q} \sup _{\eta}\left|U_{\eta}\left(\left(b_{1}-\left(b_{1}\right)_{Q^{*}}\right) f_{1}^{0},\left(b_{2}-\left(b_{2}\right)_{Q^{*}}\right) f_{2}^{\infty}\right)(z)\right|^{\delta} d z\right)^{1 / \delta}, \\
\mathfrak{T}_{43}= & \left(\frac{1}{|Q|} \int_{Q} \sup _{\eta}\left|U_{\eta}\left(\left(b_{1}-\left(b_{1}\right)_{Q^{*}}\right) f_{1}^{\infty},\left(b_{2}-\left(b_{2}\right)_{Q^{*}}\right) f_{2}^{0}\right)(z)\right|^{\delta} d z\right)^{1 / \delta}, \\
\mathfrak{T}_{44}= & \left(\frac{1}{|Q|} \int_{Q} \sup _{\eta} \mid U_{\eta}\left(\left(b_{1}-\left(b_{1}\right)_{Q^{*}}\right) f_{1}^{\infty},\left(b_{2}-\left(b_{2}\right)_{Q^{*}}\right) f_{2}^{\infty}\right)(z)\right. \\
& \left.\quad-\left.U_{\eta}\left(\left(b_{1}-\left(b_{1}\right)_{Q^{*}}\right) f_{1}^{\infty},\left(b_{2}-\left(b_{2}\right)_{Q^{*}}\right) f_{2}^{\infty}\right)(x)\right|^{\delta} d z\right)^{1 / \delta} .
\end{aligned}
$$

By the Kolmogorov inequality and by Lemma 2.2 ,

$$
\begin{aligned}
\mathfrak{T}_{41} & \lesssim\left\|U^{*}\left(\left(b_{1}-\left(b_{1}\right)_{Q^{*}}\right) f_{1}^{0},\left(b_{2}-\left(b_{2}\right)_{Q^{*}}\right) f_{2}^{0}\right)\right\|_{L^{1 / 2, \infty}(Q, d x /|Q|)} \\
& \lesssim \frac{1}{|Q|} \int_{Q}\left|\left(b_{1}-\left(b_{1}\right)_{Q^{*}}\right) f_{1}^{0}(z)\right| d z \frac{1}{|Q|} \int_{Q}\left|\left(b_{2}-\left(b_{2}\right)_{Q^{*}}\right) f_{2}^{0}(z)\right| d z \\
& \lesssim \prod_{i=1}^{2}\left\|b_{i}\right\|_{\operatorname{Lip}_{\beta_{i}}} M_{1, \beta_{i}}\left(f_{i}\right)(x) .
\end{aligned}
$$

Next, by the size condition (1.1),

$$
\begin{aligned}
\mathfrak{T}_{42} & \leq \frac{1}{|Q|} \int_{Q} \sup _{\eta}\left|U_{\eta}\left(\left(b_{1}-\left(b_{1}\right)_{Q^{*}}\right) f_{1}^{0},\left(b_{2}-\left(b_{2}\right)_{Q^{*}}\right) f_{2}^{\infty}\right)(z)\right| d z \\
& \lesssim \frac{1}{|Q|} \int_{Q} \int_{\mathbb{R}^{n}} \int_{\mathbb{R}^{n}} \sup _{\eta}\left\{\int_{0}^{\infty}\left|K_{t, \mu, \eta}\right|^{2} \frac{d t}{t}\right\}^{1 / 2}\left|\left(b_{1}\left(y_{1}\right)-\left(b_{1}\right)_{Q^{*}}\right) f_{1}^{0}\left(y_{1}\right)\right| \\
& \times \frac{1}{|Q|} \int_{Q} \int_{\mathbb{R}^{n}} \int_{\mathbb{R}^{n}} \frac{\left|\left(b_{1}\left(y_{1}\right)-\left(b_{1}\right)_{Q^{*}}\right) f_{1}^{0}\left(y_{1}\right)\right|\left|\left(b_{2}\left(y_{2}\right)-\left(b_{2}\right)_{Q^{*}}\right) f_{2}^{\infty}\left(y_{2}\right)\right| d y_{1} d y_{2}}{\left(\left|z-y_{1}\right|+\left|z-y_{2}\right|\right)^{2 n}} d z \\
& \lesssim\left\|b_{1}\right\|_{\operatorname{Lip}_{\beta_{1}}} M_{1, \beta_{1}}\left(f_{1}\right)(x)\left\|b_{2}\right\|_{\operatorname{Lip}_{\beta_{2}}} M_{1, \beta_{2}}\left(f_{2}\right)(x) .
\end{aligned}
$$

$\mathfrak{T}_{43}$ can be estimated in the same way. Finally, we estimate $\mathfrak{T}_{44}$. By using the fact $\left(\mathbb{R}^{n} \backslash Q^{*}\right)^{2} \subseteq \mathbb{R}^{2 n} \backslash\left(Q^{*}\right)^{2} \subseteq \bigcup_{k=1}^{\infty}\left(2^{k+3} \sqrt{n} Q\right)^{2} \backslash\left(2^{k+2} \sqrt{n} Q\right)^{2}$ and by Lemma 2.1 .

$$
\begin{aligned}
\mathfrak{T}_{44} \lesssim \frac{1}{|Q|} \int_{Q} \sup _{\eta} \mid U_{\eta}\left(\left(b_{1}-\left(b_{1}\right)_{Q^{*}}\right) f_{1}^{\infty},\left(b_{2}-\left(b_{2}\right)_{Q^{*}}\right) f_{2}^{\infty}\right)(z) \\
\quad-U_{\eta}\left(\left(b_{1}-\left(b_{1}\right)_{Q^{*}}\right) f_{1}^{\infty},\left(b_{2}-\left(b_{2}\right)_{Q^{*}}\right) f_{2}^{\infty}\right)(x) \mid d z \\
\lesssim \frac{1}{|Q|} \int_{Q} \int_{\left(\mathbb{R}^{n} \backslash Q^{*}\right)^{2}} \sup _{\eta}\left\{\int_{0}^{\infty}\left|K_{t, \mu, \eta}(z, \vec{y})-K_{t, \mu, \eta}\left(x_{Q}, \vec{y}\right)\right|^{2} \frac{d t}{t}\right\}^{1 / 2} \\
\quad \times \prod_{i=1}^{2}\left|\left(b_{i}\left(y_{i}\right)-\lambda_{i}\right) f_{i}^{\infty}\left(y_{i}\right)\right| d y_{1} d y_{2} d z \\
\vdots \frac{1}{|Q|} \int_{Q} \int_{\left(\mathbb{R}^{n} \backslash Q^{*}\right)^{2}} \frac{1}{\left(\left|x_{Q}-y_{1}\right|+\left|x_{Q}-y_{2}\right|\right)^{2 n}} \omega\left(\frac{\left|z-x_{Q}\right|}{\left|x_{Q}-y_{1}\right|+\left|x_{Q}-y_{2}\right|}\right)
\end{aligned}
$$




$$
\begin{gathered}
\times \prod_{i=1}^{2}\left|\left(b_{i}\left(y_{i}\right)-\lambda_{i}\right) f_{i}^{\infty}\left(y_{i}\right)\right| d y_{1} d y_{2} d z \\
+\frac{1}{|Q|} \int_{Q} \int_{\left(\mathbb{R}^{n} \backslash Q^{*}\right)^{2}} \frac{\left|z-x_{Q}\right|}{\left(\left|x_{Q}-y_{1}\right|+\left|x_{Q}-y_{2}\right|\right)^{2 n+1}} \prod_{i=1}^{2}\left|\left(b_{i}\left(y_{i}\right)-\lambda_{i}\right) f_{i}^{\infty}\left(y_{i}\right)\right| d y_{1} d y_{2} d z \\
\lesssim\left\|b_{1}\right\|_{\operatorname{Lip}_{\beta_{1}}} M_{1, \beta_{1}}\left(f_{1}\right)(x)\left\|b_{2}\right\|_{\operatorname{Lip}_{\beta_{2}}} M_{1, \beta_{2}}\left(f_{2}\right)(x) .
\end{gathered}
$$

Combining the above estimates $\mathfrak{T}_{1}, \mathfrak{T}_{2}, \mathfrak{T}_{3}$ and $\mathfrak{T}_{4}$, we obtain (2.1).

(ii) We consider the operator

$$
U_{\vec{b}}^{* 1}(\vec{f})(x)=\sup _{\eta>0}\left|\left(b(x)-b_{Q^{*}}\right) U_{\eta}\left(f_{1}, f_{2}\right)(x)-U_{\eta}\left(\left(b-b_{Q^{*}}\right) f_{1}, f_{2}\right)(x)\right|,
$$

where $b_{Q^{*}}=\frac{1}{\left|Q^{*}\right|} \int_{Q^{*}} b(y) d y$. Let $c=\sup _{\eta>0}\left|c_{\eta}\right|$, then

$$
\begin{aligned}
& \left(\left.\frac{1}{|Q|} \int_{Q}|| U_{\vec{b}}^{* 1}\left(f_{1}, f_{2}\right)(z)\right|^{\delta}-|c|^{\delta} \mid d z\right)^{1 / \delta} \\
\lesssim & \left(\frac{1}{|Q|} \int_{Q}\left|\left(b(z)-b_{Q^{*}}\right) U^{*}\left(f_{1}, f_{2}\right)(z)\right|^{\delta} d z\right)^{1 / \delta} \\
& +\left(\frac{1}{|Q|} \int_{Q} \sup _{\eta>0}\left|U_{\eta}\left(\left(b-b_{Q^{*}}\right) f_{1}, f_{2}\right)(z)-c_{\eta}\right|^{\delta} d z\right)^{1 / \delta} \\
= & : \mathfrak{L}_{1}+\mathfrak{L}_{2} .
\end{aligned}
$$

By Hölder's inequality,

$$
\mathfrak{L}_{1} \lesssim\|b\|_{\operatorname{Lip}_{\beta}} M_{\epsilon, \beta}\left(U^{*}\left(f_{1}, f_{2}\right)\right)(x) .
$$

Choose $c_{\eta}=U_{\eta}\left(\left(b-b_{Q^{*}}\right) f_{1}^{\infty}, f_{2}^{\infty}\right)(x)$. Then, $\mathfrak{L}_{2} \leq \mathfrak{L}_{21}+\mathfrak{L}_{22}+\mathfrak{L}_{23}+\mathfrak{L}_{24}$, where

$$
\begin{aligned}
\mathfrak{L}_{21} & =\left(\frac{1}{|Q|} \int_{Q}\left|U^{*}\left(\left(b-b_{Q^{*}}\right) f_{1}^{0}, f_{2}^{0}\right)(z)\right|^{\delta} d x\right)^{1 / \delta}, \\
\mathfrak{L}_{22} & =\left(\frac{1}{|Q|} \int_{Q} \sup _{\eta}\left|U_{\eta}\left(\left(b-b_{Q^{*}}\right) f_{1}^{0}, f_{2}^{\infty}\right)(z)\right|^{\delta} d z\right)^{1 / \delta}, \\
\mathfrak{L}_{23} & =\left(\frac{1}{|Q|} \int_{Q} \sup _{\eta}\left|U_{\eta}\left(\left(b-b_{Q^{*}}\right) f_{1}^{\infty}, f_{2}^{0}\right)(z)\right|^{\delta} d z\right)^{1 / \delta}, \\
\mathfrak{L}_{24} & =\left(\frac{1}{|Q|} \int_{Q} \sup _{\eta}\left|U_{\eta}\left(\left(b-b_{Q^{*}}\right) f_{1}^{\infty}, f_{2}^{\infty}\right)(z)-U_{\eta}\left(\left(b-b_{Q^{*}}\right) f_{1}^{\infty}, f_{2}^{\infty}\right)(x)\right|^{\delta} d z\right)^{1 / \delta} .
\end{aligned}
$$

By the Kolmogorov inequality and by Lemma 2.2.

$$
\begin{aligned}
& \mathfrak{L}_{21} \lesssim\left\|U^{*}\left(\left(b-b_{Q^{*}}\right) f_{1}^{0}, f_{2}^{0}\right)\right\|_{L^{1 / 2, \infty}(Q, d x /|Q|)}
\end{aligned}
$$

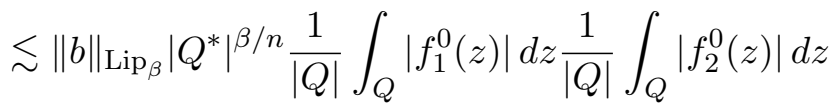

$$
\begin{aligned}
& \lesssim\|b\|_{\operatorname{Lip}_{\beta}} M_{1, \beta}\left(f_{1}\right)(x) M\left(f_{2}\right)(x) .
\end{aligned}
$$


Next, by the size condition (1.1),

$$
\begin{aligned}
\mathfrak{L}_{22} & =\left(\frac{1}{|Q|} \int_{Q} \sup _{\eta}\left|U_{\eta}\left(\left(b-b_{Q^{*}}\right) f_{1}^{0}, f_{2}^{\infty}\right)(z)\right|^{\delta} d z\right)^{1 / \delta} \\
& \lesssim \frac{1}{|Q|} \int_{Q} \sup _{\eta}\left|U_{\eta}\left(\left(b-b_{Q^{*}}\right) f_{1}^{0}, f_{2}^{\infty}\right)(z)\right| d z \\
& \lesssim \frac{1}{|Q|} \int_{Q} \int_{Q^{*}} \int_{\left(Q^{*}\right)^{c}} \sup _{\eta}\left\{\int_{0}^{\infty}\left|K_{t, \mu, \eta}\right|^{2} \frac{d t}{t}\right\}^{1 / 2}\left|\left(b\left(y_{1}\right)-b_{Q^{*}}\right) f_{1}\left(y_{1}\right)\right|\left|f_{2}\left(y_{2}\right)\right| d y_{2} d y_{1} d z \\
& \lesssim \frac{1}{|Q|} \int_{Q} \int_{Q^{*}} \int_{\left(Q^{*}\right)^{c}} \overline{\left(\left|z-y_{1}\right|+\left|z-y_{2}\right|\right)^{2 n}}\left|\left(b\left(y_{1}\right)-b_{Q^{*}}\right) f_{1}\left(y_{1}\right)\right|\left|f_{2}\left(y_{2}\right)\right| d y_{2} d y_{1} d z \\
& \lesssim\|b\|_{\operatorname{Lip}_{\beta}} M_{1, \beta}\left(f_{1}\right)(x) M\left(f_{2}\right)(x) .
\end{aligned}
$$

Similarly,

$$
\begin{aligned}
\mathfrak{L}_{23} & \lesssim \frac{1}{|Q|} \int_{Q} \sup _{\eta}\left|U_{\eta}\left(\left(b-b_{Q^{*}}\right) f_{1}^{\infty}, f_{2}^{0}\right)(z)\right| d z \\
& \lesssim \frac{1}{|Q|} \int_{Q} \int_{Q^{*}} \int_{\left(Q^{*}\right)^{c}} \sup _{\eta}\left\{\int_{0}^{\infty}\left|K_{t, \mu, \eta}\right|^{2} \frac{d t}{t}\right\}^{1 / 2}\left|\left(b\left(y_{1}\right)-b_{Q^{*}}\right) f_{1}\left(y_{1}\right)\right|\left|f_{2}\left(y_{2}\right)\right| d y_{2} d y_{1} d z \\
& \lesssim \frac{1}{|Q|} \int_{Q} \int_{Q^{*}} \int_{\left(Q^{*}\right)^{c}} \frac{1}{\left(\left|z-y_{1}\right|+\left|z-y_{2}\right|\right)^{2 n}}\left|\left(b\left(y_{1}\right)-b_{Q^{*}}\right) f_{1}\left(y_{1}\right)\right|\left|f_{2}\left(y_{2}\right)\right| d y_{2} d y_{1} d z \\
& \lesssim\|b\|_{\operatorname{Lip}_{\beta}} M_{1, \beta}\left(f_{1}\right)(x) M\left(f_{2}\right)(x) .
\end{aligned}
$$

Since $\left(\mathbb{R}^{n} \backslash Q^{*}\right)^{2} \subseteq \mathbb{R}^{2 n} \backslash\left(Q^{*}\right)^{2} \subseteq \bigcup_{k=1}^{\infty}\left(2^{k+3} \sqrt{n} Q\right)^{2} \backslash\left(2^{k+2} \sqrt{n} Q\right)^{2}$, by Lemma 2.1 .

$$
\begin{aligned}
\mathfrak{L}_{24} \lesssim & \frac{1}{|Q|} \int_{Q} \sup _{\eta}\left|U_{\eta}\left(\left(b-b_{Q^{*}}\right) f_{1}^{\infty}, f_{2}^{\infty}\right)(z)-U_{\eta}\left(\left(b-b_{Q^{*}}\right) f_{1}^{\infty}, f_{2}^{\infty}\right)(x)\right| d z \\
\lesssim & \frac{1}{|Q|} \int_{Q} \int_{\left(\mathbb{R}^{n} \backslash Q^{*}\right)^{2}} \sup _{\eta}\left\{\int_{0}^{\infty}\left|K_{t, \mu, \eta}(z, \vec{y})-K_{t, \mu, \eta}\left(x_{Q}, \vec{y}\right)\right|^{2} \frac{d t}{t}\right\}^{1 / 2} \\
& \times\left|\left(b\left(y_{1}\right)-b_{Q^{*}}\right)\right| \prod_{i=1}^{2}\left|f_{i}^{\infty}\left(y_{i}\right)\right| d y_{1} d y_{2} d z \\
\lesssim & \frac{1}{|Q|} \int_{Q} \int_{\left(\mathbb{R}^{n} \backslash Q^{*}\right)^{2}} \frac{\omega\left(\frac{\left|z-x_{Q}\right|}{\left|z-y_{1}\right|+\left|z-y_{2}\right|}\right)}{\left(\left|z-y_{1}\right|+\left|z-y_{2}\right|\right)^{2 n}}\left|\left(b\left(y_{1}\right)-b_{Q^{*}}\right)\right| \prod_{i=1}^{2}\left|f_{i}^{\infty}\left(y_{i}\right)\right| d y_{1} d y_{2} d z \\
& +\frac{1}{|Q|} \int_{Q} \int_{\left(\mathbb{R}^{n} \backslash Q^{*}\right)^{2}} \frac{\left|z-x_{Q}\right|}{\left(\left|z-y_{1}\right|+\left|z-y_{2}\right|\right)^{2 n+1}}\left|\left(b\left(y_{1}\right)-b_{Q^{*}}\right)\right| \prod_{i=1}^{2}\left|f_{i}^{\infty}\left(y_{i}\right)\right| d y_{1} d y_{2} d z \\
\lesssim & \|b\|_{\operatorname{Lip}_{\beta}} M_{1, \beta}\left(f_{1}\right)(x) M\left(f_{2}\right)(x) .
\end{aligned}
$$

Thus we finish the proof of $(2.2)$. Then Lemma 2.3 is proved.

Proof of Theorem 1.1. By using Lemma 2.2 and combining the arguments in Theorem 1.3 and [22, we can finish the proof of Theorem 1.1 without any difficulty. We omit the proof. 


\section{Proofs of Theorems 1.2 and 1.3}

In this section we prove Theorems 1.2 and 1.3 . We just consider the case $m=2$ for simplicity, our method still hold for general $m$ with little modifications.

Proof of Theorem 1.2. Theorem 1.2 will be proved if we can show

$$
\sup _{Q} \frac{1}{|Q|^{1+\beta / n-1 / p}} \int_{Q}\left|U_{\Pi \vec{b}}^{*}(\vec{f})(z)-\left(U_{\Pi \vec{b}}^{*}(\vec{f})\right)_{Q}\right| d z \lesssim\left\|b_{1}\right\|_{\dot{\lambda}_{\beta_{1}}}\left\|b_{2}\right\|_{\dot{\lambda}_{\beta_{2}}}\left\|f_{1}\right\|_{L^{p_{1}}}\left\|f_{2}\right\|_{L^{p_{2}}}
$$

We now estimate

$$
\begin{aligned}
& \frac{1}{|Q|^{1+\beta / n-1 / p}} \int_{Q}\left|U_{\Pi \vec{b}}^{*}(\vec{f})(z)-\left(U_{\Pi \vec{b}}^{*}(\vec{f})\right)_{Q}\right| d z \\
\lesssim & \frac{1}{|Q|^{1+\beta / n-1 / p}} \int_{Q}\left|U_{\Pi \vec{b}}^{*}\left(f_{1}, f_{2}\right)(z)-c\right| d z \\
\lesssim & \frac{1}{|Q|^{1+\beta / n-1 / p}} \int_{Q}\left|U_{\Pi \vec{b}}^{*}\left(f_{1}^{0}, f_{2}^{0}\right)(z)\right| d z+\frac{1}{|Q|^{1+\beta / n-1 / p}} \int_{Q}\left|U_{\Pi \vec{b}}^{*}\left(f_{1}^{0}, f_{2}^{\infty}\right)(z)-c_{1}\right| d z \\
& +\frac{1}{|Q|^{1+\beta / n-1 / p}} \int_{Q}\left|U_{\Pi \vec{b}}^{*}\left(f_{1}^{\infty}, f_{2}^{0}\right)(z)-c_{2}\right| d z+\frac{1}{|Q|^{1+\beta / n-1 / p}} \int_{Q}\left|U_{\Pi \vec{b}}^{*}\left(f_{1}^{\infty}, f_{2}^{\infty}\right)(z)-c_{3}\right| d z \\
\doteq & \mathfrak{M}_{1}+\mathfrak{M}_{2}+\mathfrak{M}_{3}+\mathfrak{M}_{4},
\end{aligned}
$$

where $c=c_{1}+c_{2}+c_{3}$, which will be determined later.

We can choose $1<q, q_{j}<\infty, q_{j}<n / \beta_{j}<p_{j}, j=1,2$ with $1 / q=1 / q_{1}+1 / q_{2}-\left(\beta_{1}+\right.$ $\left.\beta_{2}\right) / n$. By Hölder's inequality and Theorem 1.1 , we have

$$
\begin{aligned}
\mathfrak{M}_{1} & \lesssim \frac{1}{|Q|^{1+\beta / n-1 / p}}\left(\int_{Q}\left|U_{\Pi \vec{b}}^{*}\left(f_{1}^{0}, f_{2}^{0}\right)(z)\right|^{q} d z\right)^{1 / q}|Q|^{1-1 / q} \\
& \lesssim \frac{|Q|^{1-1 / q}}{|Q|^{1+\beta / n-1 / p}}\left\|f_{1}^{0}\right\|_{L^{q_{1}}}\left\|f_{2}^{0}\right\|_{L^{q_{2}}} \lesssim\left\|f_{1}\right\|_{L^{p_{1}}}\left\|f_{2}\right\|_{L^{p_{2}}} .
\end{aligned}
$$

For the second term, we take $c_{1}=U^{*}\left(\left(b_{1}-\left(b_{1}\right)_{Q^{*}}\right) f_{1}^{0},\left(b_{2}-\left(b_{2}\right)_{Q^{*}}\right) f_{2}^{\infty}\right)\left(x_{Q}\right)$. Then

$$
\begin{aligned}
\mathfrak{M}_{2} \lesssim & \frac{1}{|Q|^{1+\beta / n-1 / p}} \int_{Q} \sup _{\eta}\left(\int_{0}^{\infty} \mid \int_{Q^{*}} \int_{\mathbb{R}^{n} \backslash Q^{*}}\left(b_{1}(z)-\left(b_{1}\right)_{Q^{*}}\right)\left(b_{2}(z)-\left(b_{2}\right)_{Q^{*}}\right)\right. \\
& \left.\times\left. K_{t, \mu, \eta}\left(z, y_{1}, y_{2}\right) f_{1}\left(y_{1}\right) f_{2}\left(y_{2}\right) d y_{1} d y_{2}\right|^{2} \frac{d t}{t}\right)^{1 / 2} d z \\
+ & \frac{1}{|Q|^{1+\beta / n-1 / p}} \int_{Q} \sup _{\eta}\left(\int_{0}^{\infty} \mid \int_{Q^{*}} \int_{\mathbb{R}^{n} \backslash Q^{*}}\left(b_{1}(z)-\left(b_{1}\right)_{Q^{*}}\right)\left(b_{2}\left(y_{2}\right)-\left(b_{2}\right)_{Q^{*}}\right)\right. \\
& \left.\times\left. K_{t, \mu, \eta}\left(z, y_{1}, y_{2}\right) f_{1}\left(y_{1}\right) f_{2}\left(y_{2}\right) d y_{1} d y_{2}\right|^{2} \frac{d t}{t}\right)^{1 / 2} d z \\
+ & \frac{1}{|Q|^{1+\beta / n-1 / p}} \int_{Q} \sup _{\eta}\left(\int_{0}^{\infty} \mid \int_{Q^{*}} \int_{\mathbb{R}^{n} \backslash Q^{*}}\left(b_{1}\left(y_{1}\right)-\left(b_{1}\right)_{Q^{*}}\right)\left(b_{2}(z)-\left(b_{2}\right)_{Q^{*}}\right)\right.
\end{aligned}
$$




$$
\begin{aligned}
& \left.\times\left. K_{t, \mu, \eta}\left(z, y_{1}, y_{2}\right) f_{1}\left(y_{1}\right) f_{2}\left(y_{2}\right) d y_{1} d y_{2}\right|^{2} \frac{d t}{t}\right)^{1 / 2} d z \\
+ & \frac{1}{|Q|^{1+\beta / n-1 / p}} \int_{Q} \sup _{\eta}\left(\int_{0}^{\infty} \mid \int_{Q^{*}} \int_{\mathbb{R}^{n} \backslash Q^{*}}\left(b_{1}\left(y_{1}\right)-\left(b_{1}\right)_{Q^{*}}\right)\left(b_{2}\left(y_{2}\right)-\left(b_{2}\right)_{Q^{*}}\right)\right. \\
& \left.\quad \times\left.\left[K_{t, \mu, \eta}\left(z, y_{1}, y_{2}\right)-K_{t, \mu, \eta}\left(x_{Q}, y_{1}, y_{2}\right)\right] f_{1}\left(y_{1}\right) f_{2}\left(y_{2}\right) d y_{1} d y_{2}\right|^{2} \frac{d t}{t}\right)^{1 / 2} d z \\
\doteq & \mathfrak{M}_{21}+\mathfrak{M}_{22}+\mathfrak{M}_{23}+\mathfrak{M}_{24} .
\end{aligned}
$$

By Minkowski's inequality and the size condition (1.1), we have

$$
\begin{aligned}
& \mathfrak{M}_{21} \lesssim \frac{1}{|Q|^{1+\beta / n-1 / p}} \int_{Q} \int_{Q^{*}} \int_{\mathbb{R}^{n} \backslash Q^{*}}\left|\left(b_{1}(z)-\left(b_{1}\right)_{Q^{*}}\right)\left(b_{2}(z)-\left(b_{2}\right)_{Q^{*}}\right)\right| \\
& \times \sup _{\eta}\left(\int_{0}^{\infty}\left|K_{t, \mu, \eta}\left(z, y_{1}, y_{2}\right)\right|^{2} \frac{d t}{t}\right)^{1 / 2}\left|f_{1}\left(y_{1}\right) f_{2}\left(y_{2}\right)\right| d y_{1} d y_{2} d z \\
& \lesssim \frac{1}{|Q|^{1+\beta / n-1 / p}} \int_{Q} \int_{Q^{*}} \int_{\mathbb{R}^{n} \backslash Q^{*}}\left|\left(b_{1}(z)-\left(b_{1}\right)_{Q^{*}}\right)\left(b_{2}(z)-\left(b_{2}\right)_{Q^{*}}\right)\right| \\
& \times \frac{1}{\left(\left|z-y_{1}\right|+\left|z-y_{2}\right|\right)^{2 n}}\left|f_{1}\left(y_{1}\right) f_{2}\left(y_{2}\right)\right| d y_{1} d y_{2} d z \\
& \lesssim\left\|b_{1}\right\|_{\dot{\lambda}_{\beta_{1}}}\left\|b_{2}\right\|_{{\dot{\lambda_{\beta}}}_{\beta_{2}}}|Q|^{1 / p} \int_{Q^{*}}\left|f_{1}\left(y_{1}\right)\right| d y_{1} \sum_{k=1}^{\infty} \int_{2^{k+3} \sqrt{n} Q \backslash 2^{k+2} \sqrt{n} Q} \frac{\left|f_{2}\left(y_{2}\right)\right|}{\left|y_{2}-x_{Q}\right|^{2 n}} d y_{2} \\
& \lesssim\left\|b_{1}\right\|_{\dot{\Lambda}_{\beta_{1}}}\left\|b_{2}\right\|_{{\dot{\lambda}}_{\beta_{2}}}\left\|f_{1}\right\|_{L^{p_{1}}}\left\|f_{2}\right\|_{L^{p_{2}}} \sum_{k=1}^{\infty} 2^{k n\left(-1-1 / p_{2}\right)} \\
& \lesssim\left\|b_{1}\right\|_{{\dot{\lambda}}_{1}}\left\|b_{2}\right\|_{{\dot{\lambda}}_{\beta_{2}}}\left\|f_{1}\right\|_{L^{p_{1}}}\left\|f_{2}\right\|_{L^{p_{2}}}
\end{aligned}
$$

Similarly,

$$
\mathfrak{M}_{22}+\mathfrak{M}_{23} \lesssim\left\|b_{1}\right\|_{{\dot{\beta}}_{1}}\left\|b_{2}\right\|_{{\dot{\lambda_{\beta}}}_{\beta_{2}}}\left\|f_{1}\right\|_{L^{p_{1}}}\left\|f_{2}\right\|_{L^{p_{2}}}
$$

By Minkowski's inequality and Lemma 2.1, we have

$\mathfrak{M}_{24}$

$$
\begin{aligned}
& \lesssim \frac{1}{|Q|^{1+\beta / n-1 / p}} \int_{Q} \int_{Q^{*}} \int_{\mathbb{R}^{n} \backslash Q^{*}}\left|\left(b_{1}(z)-\left(b_{1}\right)_{Q^{*}}\right)\left(b_{2}\left(y_{2}\right)-\left(b_{2}\right)_{Q^{*}}\right)\right| \\
& \quad \times \sup _{\eta}\left(\int_{0}^{\infty}\left|K_{t, \mu, \eta}\left(z, y_{1}, y_{2}\right)-K_{t, \mu, \eta}\left(x_{Q}, y_{1}, y_{2}\right)\right|^{2} \frac{d t}{t}\right)^{1 / 2}\left|f_{1}\left(y_{1}\right) f_{2}\left(y_{2}\right)\right| d y_{1} d y_{2} d z \\
& \lesssim\left\|b_{1}\right\|_{\dot{\lambda}_{\beta_{1}}}\left\|b_{2}\right\|_{{\dot{\lambda}}_{\beta_{2}}} \frac{1}{|Q|^{1+\beta / n-1 / p}} \int_{Q} \int_{Q^{*}} \int_{\mathbb{R}^{n} \backslash Q^{*}}\left|\left(b_{1}\left(y_{1}\right)-\lambda_{1}\right)\left(b_{2}\left(y_{2}\right)-\lambda_{2}\right)\right| \\
& \quad \times\left(\frac{\omega\left(\frac{\left|z-x_{Q}\right|}{\left|z-y_{1}\right|+\left|z-y_{2}\right|}\right)}{\left(\left|z-y_{1}\right|+\left|z-y_{2}\right|\right)^{2 n}}+\frac{\left|z-x_{Q}\right|}{\left(\left|x-y_{1}\right|+\left|x-y_{2}\right|\right)^{2 n+1}}\right)\left|f_{1}\left(y_{1}\right) f_{2}\left(y_{2}\right)\right| d y_{1} d y_{2} d z \\
& \lesssim\left\|b_{1}\right\|_{\dot{\lambda}_{\beta_{1}}}\left\|b_{2}\right\|_{\dot{\lambda}_{\beta_{2}}} \frac{1}{|Q|^{1+\beta_{2} / n-1 / p}} \int_{Q} \int_{Q^{*}} \int_{\mathbb{R}^{n} \backslash Q^{*}}
\end{aligned}
$$




$$
\begin{aligned}
& \times\left(\frac{\omega\left(\frac{\left|z-x_{Q}\right|}{\left|x_{Q}-y_{2}\right|}\right)}{\left(\left|z-y_{1}\right|+\left|z-y_{2}\right|\right)^{2 n-\beta_{2}}}+\frac{2^{-k}}{\left(\left|z-y_{1}\right|+\left|z-y_{2}\right|\right)^{2 n-\beta_{2}}}\right)\left|f_{1}\left(y_{1}\right) f_{2}\left(y_{2}\right)\right| d y_{1} d y_{2} d z \\
& \lesssim \frac{\left\|b_{1}\right\|_{\dot{\Lambda}_{\beta_{1}}}\left\|b_{2}\right\|_{\dot{\Lambda}_{\beta_{2}}}}{|Q|^{\beta_{2} / n-1 / p}} \int_{Q^{*}}\left|f_{1}\left(y_{1}\right)\right| d y_{1} \sum_{k=1}^{\infty} \frac{\omega\left(2^{-k}\right)+2^{-k}}{\left|2^{k+3} \sqrt{n} Q\right|^{2-\beta_{2} / n}} \int_{2^{k+3} \sqrt{n} Q \backslash 2^{k+2} \sqrt{n} Q}\left|f_{2}\left(y_{2}\right)\right| d y_{2} \\
& \lesssim\left\|b_{1}\right\|_{\dot{\lambda}_{\beta_{1}}}\left\|b_{2}\right\|_{{\dot{\lambda}}_{\beta_{2}}}\left\|f_{1}\right\|_{L^{p_{1}}}\left\|f_{2}\right\|_{L^{p_{2}}} \sum_{k=1}^{\infty}\left(\omega\left(2^{-k}\right)+2^{-k}\right) 2^{-k n\left(1-\beta_{2} / n+1 / p_{2}\right)} \\
& \lesssim\left\|b_{1}\right\|_{{\dot{\lambda}}_{\beta_{1}}}\left\|b_{2}\right\|_{{\dot{\lambda}}_{\beta_{2}}}\left\|f_{1}\right\|_{L^{p_{1}}}\left\|f_{2}\right\|_{L^{p_{2}}},
\end{aligned}
$$

where we have used the fact $1-\beta_{2} / n+1 / p_{2}>0$. Thus,

$$
\mathfrak{M}_{2} \lesssim\left\|b_{1}\right\|_{{\dot{\lambda_{\beta}}}_{\beta_{1}}}\left\|b_{2}\right\|_{{\dot{\lambda_{\beta}}}_{\beta_{2}}}\left\|f_{1}\right\|_{L^{p_{1}}}\left\|f_{2}\right\|_{L^{p_{2}}}
$$

Similarly,

$$
\mathfrak{M}_{3} \lesssim\left\|b_{1}\right\|_{{\dot{\lambda_{\beta}}}_{\beta_{1}}}\left\|b_{2}\right\|_{{\dot{\lambda_{\beta}}}_{\beta_{2}}}\left\|f_{1}\right\|_{L^{p_{1}}}\left\|f_{2}\right\|_{L^{p_{2}}}
$$

We deal with $\mathfrak{M}_{4}$ as follows:

$$
\begin{aligned}
\mathfrak{M}_{4} \leq & \frac{C}{|Q|^{1+\beta / n-1 / p}} \int_{Q} \sup _{\eta}\left(\int_{0}^{\infty} \mid \int_{\left(\mathbb{R}^{n} \backslash Q^{*}\right)^{2}}\left(b_{1}(z)-\left(b_{1}\right)_{Q^{*}}\right)\left(b_{2}(z)-\left(b_{2}\right)_{Q^{*}}\right)\right. \\
& \left.\times\left. K_{t, \mu, \eta}\left(z, y_{1}, y_{2}\right) f_{1}\left(y_{1}\right) f_{2}\left(y_{2}\right) d y_{1} d y_{2}\right|^{2} \frac{d t}{t}\right)^{1 / 2} d z \\
+ & \frac{C}{|Q|^{1+\beta / n-1 / p}} \int_{Q} \sup _{\eta}\left(\int_{0}^{\infty} \mid \int_{\left(\mathbb{R}^{n} \backslash Q^{*}\right)^{2}}\left(b_{1}(z)-\left(b_{1}\right)_{Q^{*}}\right)\left(b_{2}\left(y_{2}\right)-\left(b_{2}\right)_{Q^{*}}\right)\right. \\
& \left.\times\left.\left[K_{t, \mu, \eta}\left(z, y_{1}, y_{2}\right)-K_{t, \mu, \eta}\left(x_{Q}, y_{1}, y_{2}\right)\right] f_{1}\left(y_{1}\right) f_{2}\left(y_{2}\right) d y_{1} d y_{2}\right|^{2} \frac{d t}{t}\right)^{1 / 2} d z \\
+ & \frac{C}{|Q|^{1+\beta / n-1 / p}} \int_{Q} \sup _{\eta}\left(\int_{0}^{\infty} \mid \int_{\left(\mathbb{R}^{n} \backslash Q^{*}\right)^{2}}\left(b_{1}\left(y_{1}\right)-\left(b_{1}\right)_{Q^{*}}\right)\left(b_{2}(z)-\left(b_{2}\right)_{Q^{*}}\right)\right. \\
& \left.\times\left.\left[K_{t, \mu, \eta}\left(z, y_{1}, y_{2}\right)-K_{t, \mu, \eta}\left(x_{Q}, y_{1}, y_{2}\right)\right] f_{1}\left(y_{1}\right) f_{2}\left(y_{2}\right) d y_{1} d y_{2}\right|^{2} \frac{d t}{t}\right)^{1 / 2} d z \\
+ & \frac{C}{|Q|^{1+\beta / n-1 / p}} \int_{Q} \sup _{\eta}\left(\int_{0}^{\infty} \mid \int_{\left(\mathbb{R}^{n} \backslash Q^{*}\right)^{2}}\left(b_{1}\left(y_{1}\right)-\left(b_{1}\right)_{Q^{*}}\right)\left(b_{2}\left(y_{2}\right)-\left(b_{2}\right)_{Q^{*}}\right)\right. \\
& \left.\times\left.\left[K_{t, \mu, \eta}\left(z, y_{1}, y_{2}\right)-K_{t, \mu, \eta}\left(x_{Q}, y_{1}, y_{2}\right)\right] f_{1}\left(y_{1}\right) f_{2}\left(y_{2}\right) d y_{1} d y_{2}\right|^{2} \frac{d t}{t}\right)^{1 / 2} d z \\
\doteq & \mathfrak{M}_{41}+\mathfrak{M}_{42}+\mathfrak{M}_{43}+\mathfrak{M}_{44} \cdot
\end{aligned}
$$

By Minkowski's inequality and the size condition (1.1), we have

$$
\begin{aligned}
\mathfrak{M}_{41} \lesssim & \frac{1}{|Q|^{1+\beta / n-1 / p}} \int_{Q} \int_{\left(\mathbb{R}^{n} \backslash Q^{*}\right)^{2}}\left|\left(b_{1}(z)-\left(b_{1}\right)_{Q^{*}}\right)\left(b_{2}(z)-\left(b_{2}\right)_{Q^{*}}\right)\right| \\
& \quad \times \sup _{\eta}\left(\int_{0}^{\infty}\left|K_{t, \mu, \eta}\left(z, y_{1}, y_{2}\right)\right|^{2} \frac{d t}{t}\right)^{1 / 2}\left|f_{1}\left(y_{1}\right) f_{2}\left(y_{2}\right)\right| d y_{1} d y_{2} d z
\end{aligned}
$$




$$
\begin{aligned}
& \lesssim \frac{1}{|Q|^{1+\beta / n-1 / p}} \int_{Q} \int_{\left(\mathbb{R}^{n} \backslash Q^{*}\right)^{2}}\left|\left(b_{1}(z)-\left(b_{1}\right)_{Q^{*}}\right)\left(b_{2}(z)-\left(b_{2}\right)_{Q^{*}}\right)\right| \\
& \quad \times \frac{1}{\left(\left|z-y_{1}\right|+\left|z-y_{2}\right|\right)^{2 n}}\left|f_{1}\left(y_{1}\right) f_{2}\left(y_{2}\right)\right| d y_{1} d y_{2} d z \\
& \lesssim\left\|b_{1}\right\|_{\dot{\lambda}_{\beta_{1}}}\left\|b_{2}\right\|_{\dot{\lambda}_{\beta_{2}}}|Q|^{1 / p} \sum_{k=1}^{\infty} \int_{2^{k+3} \sqrt{n} Q \backslash 2^{k+2} \sqrt{n} Q} \frac{\left|f_{1}\left(y_{1}\right)\right|}{\left|y_{1}-x_{Q}\right|^{n}} d y_{1} \\
& \quad \times \sum_{k=1}^{\infty} \int_{2^{k+3} \sqrt{n} Q \backslash 2^{k+2} \sqrt{n} Q} \frac{\left|f_{2}\left(y_{2}\right)\right|}{\left|y_{2}-x_{Q}\right|^{n}} d y_{2} \\
& \lesssim\left\|b_{1}\right\|_{\dot{\lambda}_{\beta_{1}}}\left\|b_{2}\right\|_{\dot{\lambda}_{\beta_{2}}}\left\|f_{1}\right\|_{L^{p_{1}}}\left\|f_{2}\right\|_{L^{p_{2}}|Q|^{1 / p}} \sum_{k=1}^{\infty}\left|2^{k+3} \sqrt{n} Q\right|^{-1 / p_{1}} \sum_{k=1}^{\infty}\left|2^{k+3} \sqrt{n} Q\right|^{-1 / p_{2}} \\
& \lesssim\left\|b_{1}\right\|_{\dot{\lambda}_{\beta_{1}}}\left\|b_{2}\right\|_{\dot{\lambda}_{\beta_{2}}}\left\|f_{1}\right\|_{L^{p_{1}}}\left\|f_{2}\right\|_{L^{p_{2}}} .
\end{aligned}
$$

By Minkowski's inequality and Lemma 2.1, we have

$$
\begin{aligned}
& \mathfrak{M}_{42} \lesssim \frac{1}{|Q|^{1+\beta / n-1 / p}} \int_{Q} \int_{\left(\mathbb{R}^{n} \backslash Q^{*}\right)^{2}}\left|\left(b_{1}(z)-\left(b_{1}\right)_{Q^{*}}\right)\left(b_{2}\left(y_{2}\right)-\left(b_{2}\right)_{Q^{*}}\right)\right| \\
& \times \sup _{\eta}\left(\int_{0}^{\infty}\left|K_{t, \mu, \eta}\left(z, y_{1}, y_{2}\right)-K_{t, \mu, \eta}\left(x_{Q}, y_{1}, y_{2}\right)\right|^{2} \frac{d t}{t}\right)^{1 / 2}\left|f_{1}\left(y_{1}\right) f_{2}\left(y_{2}\right)\right| d y_{1} d y_{2} d z \\
& \lesssim \frac{\left\|b_{1}\right\|_{\dot{\lambda}_{\beta_{1}}}\left\|b_{2}\right\|_{\dot{\lambda}_{\beta_{2}}}}{|Q|^{\beta_{2} / n-1 / p}} \sum_{k=1}^{\infty} \int_{2^{k+3} \sqrt{n} Q \backslash 2^{k+2} \sqrt{n} Q} \frac{\left|f_{1}\left(y_{1}\right)\right|}{\left|y_{1}-x_{Q}\right|^{n}} d y_{1} \\
& \times \sum_{k=1}^{\infty} \int_{2^{k+3} \sqrt{n} Q \backslash 2^{k+2} \sqrt{n} Q} \frac{\left|f_{2}\left(y_{2}\right)\right|\left(\omega\left(2^{-k}\right)+\frac{\left|z-x_{Q}\right|}{\left|x_{Q}-y_{1}\right|+\left|x_{Q}-y_{2}\right|}\right)}{\left|y_{2}-x_{Q}\right|^{n-\beta_{2}}} d y_{2} \\
& \lesssim \frac{\left\|b_{1}\right\|_{{\dot{\lambda}}_{\beta_{1}}}\left\|b_{2}\right\|_{\dot{\Lambda}_{\beta_{2}}}}{|Q|^{\beta_{2} / n-1 / p}} \sum_{k=1}^{\infty} \frac{1}{\left|2^{k+3} \sqrt{n} Q\right|} \int_{2^{k+3} \sqrt{n} Q} f_{1}\left(y_{1}\right) d y_{1} \\
& \times \sum_{k=1}^{\infty}\left(\omega\left(2^{-k}\right)+2^{-k}\right) \frac{1}{\left|2^{k+3} \sqrt{n} Q\right|^{1-\beta_{2} / n}} \int_{2^{k+3} \sqrt{n} Q} f_{2}\left(y_{2}\right) d y_{2} \\
& \lesssim\left\|b_{1}\right\|_{{\dot{\lambda}}_{1}}\left\|b_{2}\right\|_{{\dot{\lambda}}_{\beta_{2}}}\left\|f_{1}\right\|_{L^{p_{1}}}\left\|f_{2}\right\|_{L^{p_{2}}} \sum_{k=1}^{\infty}\left(\omega\left(2^{-k}\right)+2^{-k}\right) 2^{k n\left(\beta_{2} / n-1 / p\right)} \\
& \lesssim\left\|b_{1}\right\|_{{\dot{\lambda_{\beta}}}_{\beta_{1}}}\left\|b_{2}\right\|_{{\dot{\lambda_{\beta}}}_{\beta_{2}}}\left\|f_{1}\right\|_{L^{p_{1}}}\left\|f_{2}\right\|_{L^{p_{2}}}
\end{aligned}
$$

where we have used the assumption (1.3) and the fact $0<\beta-n / p<1$. Similarly,

$$
\mathfrak{M}_{43} \lesssim\left\|b_{1}\right\|_{\dot{\lambda}_{\beta_{1}}}\left\|b_{2}\right\|_{{\dot{\lambda}}_{2}}\left\|f_{1}\right\|_{L^{p_{1}}}\left\|f_{2}\right\|_{L^{p_{2}}}
$$

Now we estimate $\mathfrak{M}_{44}$ :

$$
\mathfrak{M}_{44} \lesssim \frac{1}{|Q|^{1+\beta / n-1 / p}} \int_{Q} \int_{\left(\mathbb{R}^{n} \backslash Q^{*}\right)^{2}}\left|\left(b_{1}\left(y_{1}\right)-\left(b_{1}\right)_{Q^{*}}\right)\left(b_{2}\left(y_{2}\right)-\left(b_{2}\right)_{Q^{*}}\right)\right|
$$




$$
\begin{aligned}
& \times \sup _{\eta}\left(\int_{0}^{\infty}\left|K_{t, \mu, \eta}\left(z, y_{1}, y_{2}\right)-K_{t, \mu, \eta}\left(x_{Q}, y_{1}, y_{2}\right)\right|^{2} \frac{d t}{t}\right)^{1 / 2}\left|f_{1}\left(y_{1}\right) f_{2}\left(y_{2}\right)\right| d y_{1} d y_{2} d z \\
& \lesssim \frac{\left\|b_{1}\right\|_{\dot{\lambda}_{\beta_{1}}}\left\|b_{2}\right\|_{\dot{\lambda}_{\beta_{2}}}}{|Q|^{1+\beta / n-1 / p}} \int_{Q} \sum_{k=1}^{\infty} \int_{\left(2^{k+3} \sqrt{n} Q\right)^{2} \backslash\left(2^{k+2} \sqrt{n} Q\right)^{2}} \frac{\left|f_{1}\left(y_{1}\right)\right|}{\left|y_{2}-x_{Q}\right|^{2 n-\beta_{1}-\beta_{2}}} \\
& \times\left(\omega\left(\frac{\left|z-x_{Q}\right|}{\left|y_{2}-x_{Q}\right|}\right)+\frac{\left|z-x_{Q}\right|}{\left|x_{Q}-y_{1}\right|+\left|x_{Q}-y_{2}\right|}\right) d y_{1} d y_{2} d z \\
& \lesssim\left\|b_{1}\right\|_{{\dot{\lambda}}_{1}}\left\|b_{2}\right\|_{{\dot{\lambda_{\beta}}}_{\beta_{2}}}\left\|f_{1}\right\|_{L^{p_{1}}}\left\|f_{2}\right\|_{L^{p_{2}}} \sum_{k=1}^{\infty}\left(\omega\left(2^{-k}\right)+2^{-k}\right) 2^{k n(\beta / n-1 / p)} \\
& \lesssim\left\|b_{1}\right\|_{{\dot{\lambda_{\beta}}}_{\beta_{1}}}\left\|b_{2}\right\|_{{\dot{\lambda_{\beta_{2}}}}_{\beta_{2}}}\left\|f_{1}\right\|_{L^{p_{1}}}\left\|f_{2}\right\|_{L^{p_{2}}}
\end{aligned}
$$

Combing the estimates for $\mathfrak{M}_{1}, \mathfrak{M}_{2}, \mathfrak{M}_{3}, \mathfrak{M}_{4}$, we get 3.1 . Thus the proof of Theorem 1.2 is completed.

Proof of Theorem 1.3. Let $c=c_{1}+c_{2}+c_{3}$, which will be determined later. Then

$$
\begin{aligned}
& \frac{1}{|Q|^{1+\beta / n}} \int_{Q}\left|U_{\Pi \vec{b}}^{*}(\vec{f})(z)-\left(U_{\Pi \vec{b}}^{*}(\vec{f})\right)_{Q}\right| d z \\
\lesssim & \frac{1}{|Q|^{1+\beta / n}} \int_{Q}\left|U_{\Pi \vec{b}}^{*}\left(f_{1}, f_{2}\right)(z)-c\right| d z \\
\lesssim & \frac{1}{|Q|^{1+\beta / n}} \int_{Q}\left|\left(b_{1}(z)-\left(b_{1}\right)_{Q^{*}}\right)\left(b_{2}(z)-\left(b_{2}\right)_{Q^{*}}\right) U^{*}\left(f_{1}, f_{2}\right)(z)\right| d z \\
& +\frac{1}{|Q|^{1+\beta / n}} \int_{Q}\left|\left(b_{2}(z)-\left(b_{2}\right)_{Q^{*}}\right) U_{\vec{b}}^{*, 1}\left(f_{1}, f_{2}\right)(z)-c_{1}\right| d z \\
& +\frac{1}{|Q|^{1+\beta / n}} \int_{Q}\left|\left(b_{1}(z)-\left(b_{1}\right)_{Q^{*}}\right) U_{\vec{b}}^{*, 2}\left(f_{1}, f_{2}\right)(z)-c_{2}\right| d z \\
& +\frac{1}{|Q|^{1+\beta / n}} \int_{Q}\left|U^{*}\left(\left(b_{1}-\left(b_{1}\right)_{Q^{*}}\right) f_{1},\left(b_{2}-\left(b_{2}\right)_{Q^{*}}\right) f_{2}\right)(z)-c_{3}\right| d z \\
\doteq & \mathfrak{N}_{1}+\mathfrak{N}_{2}+\mathfrak{N}_{3}+\mathfrak{N}_{4} .
\end{aligned}
$$

For $1<r<p$, by the Hölder inequality, one has

$$
\mathfrak{N}_{1} \lesssim\left\|b_{1}\right\|_{{\dot{\lambda_{\beta}}}_{\beta_{1}}}\left\|b_{2}\right\|_{{\dot{\lambda}}_{2}} M_{r}\left(U^{*}\left(f_{1}, f_{2}\right)\right)(x)
$$

In what follows we just give the estimate for $\mathfrak{N}_{2}$, since $\mathfrak{N}_{3}$ and $\mathfrak{N}_{4}$ can be estimated in the same way. Let $c_{1}^{\prime}=\left\|b_{2}\right\|_{{\dot{\beta}}_{\beta_{2}}}|Q|^{\beta_{2} / n}(A+B+C)$, where

$$
\begin{aligned}
& A=\sup _{\eta}\left(\int_{0}^{\infty}\left|\int_{\left(\mathbb{R}^{n}\right)^{m}}\left(b_{1}\left(y_{1}\right)-\left(b_{1}\right)_{Q^{*}}\right) K_{t, \mu, \eta}\left(x, y_{1}, y_{2}\right) f_{1}^{\infty}\left(y_{1}\right) f_{2}^{0}\left(y_{2}\right) d y_{1} d y_{2}\right|^{2} \frac{d t}{t}\right)^{1 / 2}, \\
& B=\sup _{\eta}\left(\int_{0}^{\infty}\left|\int_{\left(\mathbb{R}^{n}\right)^{m}}\left(b_{1}\left(y_{1}\right)-\left(b_{1}\right)_{Q^{*}}\right) K_{t, \mu, \eta}\left(x, y_{1}, y_{2}\right) f_{1}^{0}\left(y_{1}\right) f_{2}^{\infty}\left(y_{2}\right) d y_{1} d y_{2}\right|^{2} \frac{d t}{t}\right)^{1 / 2}, \\
& C=\sup _{\eta}\left(\int_{0}^{\infty}\left|\int_{\left(\mathbb{R}^{n}\right)^{m}}\left(b_{1}\left(y_{1}\right)-\left(b_{1}\right)_{Q^{*}}\right) K_{t, \mu, \eta}\left(x, y_{1}, y_{2}\right) f_{1}^{\infty}\left(y_{1}\right) f_{2}^{\infty}\left(y_{2}\right) d y_{1} d y_{2}\right|^{2} \frac{d t}{t}\right)^{1 / 2} .
\end{aligned}
$$


Observe that

$$
\begin{aligned}
& U_{\vec{b}}^{*, 1}\left(f_{1}, f_{2}\right)(z) \\
\leq & \left|\left(b_{1}(z)-\left(b_{1}\right) Q_{Q^{*}}\right)\right| U^{*}\left(f_{1}, f_{2}\right)(z)+U^{*}\left(\left(b_{1}-\left(b_{1}\right)_{Q^{*}}\right) f_{1}^{0}, f_{2}^{0}\right)(z) \\
& +\sup _{\eta}\left(\int_{0}^{\infty}\left|\int_{\left(\mathbb{R}^{n}\right)^{m}}\left(b_{1}\left(y_{1}\right)-\left(b_{1}\right)_{Q^{*}}\right) K_{t, \mu, \eta}\left(x, y_{1}, y_{2}\right) f_{1}^{\infty}\left(y_{1}\right) f_{2}^{0}\left(y_{2}\right) d y_{1} d y_{2}\right|^{2} \frac{d t}{t}\right)^{1 / 2} \\
& +\sup _{\eta}\left(\int_{0}^{\infty}\left|\int_{\left(\mathbb{R}^{n}\right)^{m}}\left(b_{1}\left(y_{1}\right)-\left(b_{1}\right)_{Q^{*}}\right) K_{t, \mu, \eta}\left(x, y_{1}, y_{2}\right) f_{1}^{0}\left(y_{1}\right) f_{2}^{\infty}\left(y_{2}\right) d y_{1} d y_{2}\right|^{2} \frac{d t}{t}\right)^{1 / 2} \\
& +\sup _{\eta}\left(\int_{0}^{\infty}\left|\int_{\left(\mathbb{R}^{n}\right)^{m}}\left(b_{1}\left(y_{1}\right)-\left(b_{1}\right)_{Q^{*}}\right) K_{t, \mu, \eta}\left(x, y_{1}, y_{2}\right) f_{1}^{\infty}\left(y_{1}\right) f_{2}^{\infty}\left(y_{2}\right) d y_{1} d y_{2}\right|^{2} \frac{d t}{t}\right)^{1 / 2} .
\end{aligned}
$$

From this,

$$
\begin{aligned}
\mathfrak{N}_{2} \lesssim & \left.\frac{1}{|Q|^{1+\beta / n}} \int_{Q}\left|\left\|b_{2}\right\|_{\dot{\lambda}_{\beta_{2}}}\right| Q\right|^{\beta_{2} / n} U_{\vec{b}}^{*, 1}\left(f_{1}, f_{2}\right)(z)-c_{1}^{\prime} \mid d z \\
\lesssim & \frac{\left\|b_{2}\right\|_{\dot{\lambda}_{\beta_{2}}}}{|Q|^{1+\beta_{1} / n}} \int_{Q}\left|\left(b_{1}(z)-\left(b_{1}\right)_{Q^{*}}\right)\right| U^{*}\left(f_{1}, f_{2}\right)(z) d z \\
+ & \frac{\left\|b_{2}\right\|_{\dot{\lambda}_{\beta_{2}}}}{|Q|^{1+\beta_{1} / n}} \int_{Q} U^{*}\left(\left(b_{1}-\left(b_{1}\right)_{Q^{*}}\right) f_{1}^{0}, f_{2}^{0}\right)(z) d z \\
+ & \frac{C\left\|b_{2}\right\|_{\dot{\lambda}_{\beta_{2}}}}{|Q|^{1+\beta_{1} / n}} \int_{Q}\left(\int_{0}^{\infty} \mid \int_{\left(\mathbb{R}^{n}\right)^{m}}\left(b_{1}\left(y_{1}\right)-\left(b_{1}\right)_{Q^{*}}\right)\right. \\
& \left.\times\left.\left[K_{t, \mu, \eta}\left(z, y_{1}, y_{2}\right)-K_{t, \mu, \eta}\left(x_{Q}, y_{1}, y_{2}\right)\right] f_{1}^{0}\left(y_{1}\right) f_{2}^{\infty}\left(y_{2}\right) d y_{1} d y_{2}\right|^{2} \frac{d t}{t}\right)^{1 / 2} d z \\
+ & \frac{C\left\|b_{2}\right\|_{\dot{\lambda}_{\beta_{2}}}}{|Q|^{1+\beta_{1} / n}} \int_{Q}\left(\int_{0}^{\infty} \mid \int_{\left(\mathbb{R}^{n}\right)^{m}}\left(b_{1}\left(y_{1}\right)-\left(b_{1}\right)_{Q^{*}}\right)\right. \\
& \left.\times\left.\left[K_{t, \mu, \eta}\left(z, y_{1}, y_{2}\right)-K_{t, \mu, \eta}\left(x_{Q}, y_{1}, y_{2}\right)\right] f_{1}^{\infty}\left(y_{1}\right) f_{2}^{0}\left(y_{2}\right) d y_{1} d y_{2}\right|^{2} \frac{d t}{t}\right)^{1 / 2} d z \\
+ & \frac{C\left\|b_{2}\right\|_{\dot{\lambda}_{\beta_{2}}}}{|Q|^{1+\beta_{1} / n}} \int_{Q}\left(\int_{0}^{\infty} \mid \int_{\left(\mathbb{R}^{n}\right)^{m}}\left(b_{1}\left(y_{1}\right)-\left(b_{1}\right)_{Q^{*}}\right)\right. \\
& \left.\times\left.\left[K_{t, \mu, \eta}\left(z, y_{1}, y_{2}\right)-K_{t, \mu, \eta}\left(x_{Q}, y_{1}, y_{2}\right)\right] f_{1}^{\infty}\left(y_{1}\right) f_{2}^{\infty}\left(y_{2}\right) d y_{1} d y_{2}\right|^{2} \frac{d t}{t}\right)^{1 / 2} d z \\
\doteq & \mathfrak{N}_{21}+\mathfrak{N}_{22}+\mathfrak{N}_{23}+\mathfrak{N}_{24}+\mathfrak{N}_{25} .
\end{aligned}
$$

By the Hölder inequality, $\mathfrak{N}_{21}$ can be controlled by a constant times

$$
\begin{aligned}
& \left\|b_{2}\right\|_{\dot{\wedge}_{\beta_{2}}}\left(\frac{1}{|Q|^{r^{\prime} \beta_{1} / n+1}} \int_{Q}\left|b_{1}(z)-\left(b_{1}\right)_{Q^{*}}\right|^{r^{\prime}} d z\right)^{1 / r^{\prime}}\left(\frac{1}{|Q|} \int_{Q}\left|U^{*}\left(f_{1}, f_{2}\right)(z)\right|^{r} d z\right)^{1 / r} \\
& \lesssim\left\|b_{1}\right\|_{{\dot{\aleph_{\beta}}}_{\beta_{1}}}\left\|b_{2}\right\|_{{\dot{\lambda_{\beta_{2}}}}} M_{r}\left(U^{*}\left(f_{1}, f_{2}\right)\right)(x) .
\end{aligned}
$$


Take $1<q_{1}<p_{1}, 1<q_{2}<p_{2}$ and $1<q<\infty$, such that $1 / q=1 / q_{1}+1 / q_{2}$, then by Hölder inequality and by Lemma 2.2 ,

$$
\begin{aligned}
\mathfrak{N}_{22} & \lesssim \frac{\left\|b_{2}\right\|_{\dot{\wedge}_{\beta_{2}}}}{|Q|^{\beta_{1} / n+1 / q}}\left(\int_{Q}\left|U^{*}\left(\left(b_{1}-\left(b_{1}\right)_{Q^{*}}\right) f_{1}^{0}, f_{2}^{0}\right)(z)\right|^{q} d z\right)^{1 / q} \\
& \lesssim \frac{\left\|b_{2}\right\|_{\dot{\wedge}_{\beta_{2}}}}{|Q|^{\beta_{1} / n+1 / q}}\left\|\left(b_{1}-\left(b_{1}\right)_{Q^{*}}\right) f_{1}^{0}\right\|_{L^{q_{1}}}\left\|f_{2}^{0}\right\|_{L^{q_{2}}} \\
& \lesssim \frac{\left\|b_{1}\right\|_{\dot{\wedge}_{\beta_{1}}}\left\|b_{2}\right\|_{\dot{\lambda}_{\beta_{2}}}\left\|f_{1}^{0}\right\|_{L^{q_{1}}}\left\|f_{2}^{0}\right\|_{L^{q_{2}}}}{|Q|^{1 / q}} \\
& \lesssim\left\|b_{1}\right\|_{{\dot{\lambda_{\beta}}}_{\beta_{1}}}\left\|b_{2}\right\|_{{\dot{\lambda_{\beta}}}_{\beta_{2}}} M_{q_{1}}\left(f_{1}\right)(x) M_{q_{2}}\left(f_{2}\right)(x)
\end{aligned}
$$

For $y_{2} \in\left(Q^{*}\right)^{c},\left|y_{2}-x_{Q}\right| \sim\left|y_{2}-z\right|,\left|z-x_{Q}\right| \leq \frac{\left|y_{2}-z\right|}{3} \leq \frac{1}{3} \max \left\{\left|z-y_{1}\right|,\left|z-y_{2}\right|\right\}$, then by Minkowski's inequality and by Lemma 2.1.

$$
\begin{aligned}
& \mathfrak{N}_{23} \\
& \lesssim \frac{\left\|b_{2}\right\|_{\dot{\lambda}_{\beta_{2}}}}{|Q|^{1+\beta_{1} / n}} \int_{Q} \sup _{\eta} \int_{\left(\mathbb{R}^{n}\right)^{2}}\left|\left(b_{1}\left(y_{1}\right)-\left(b_{1}\right)_{Q^{*}}\right)\right| \\
& \times\left(\int_{0}^{\infty}\left|K_{t, \mu, \eta}\left(z, y_{1}, y_{2}\right)-K_{t, \mu, \eta}\left(x_{Q}, y_{1}, y_{2}\right)\right|^{2} \frac{d t}{t}\right)^{1 / 2}\left|f_{1}^{0}\left(y_{1}\right) f_{2}^{\infty}\left(y_{2}\right)\right| d y_{1} d y_{2} d z \\
& \lesssim \frac{\left\|b_{1}\right\|_{\dot{\wedge}_{\beta_{1}}}\left\|b_{2}\right\|_{\dot{\lambda}_{\beta_{2}}}}{|Q|} \int_{Q} \int_{\left(\mathbb{R}^{n}\right)^{2}} \frac{\left|f_{1}^{0}\left(y_{1}\right) f_{2}^{\infty}\left(y_{2}\right)\right|}{\left(\left|z-y_{1}\right|+\left|z-y_{2}\right|\right)^{2 n}} \\
& \times\left(\omega\left(\frac{\left|z-x_{Q}\right|}{\left|z-y_{1}\right|+\left|z-y_{2}\right|}\right)+\frac{\left|z-x_{Q}\right|}{\left|z-y_{1}\right|+\left|z-y_{2}\right|}\right) d y_{1} d y_{2} d z \\
& \lesssim \frac{\left\|b_{1}\right\|{\dot{\dot{\lambda}_{\beta}}}_{\beta_{1}}\left\|b_{2}\right\|_{\dot{\lambda}_{\beta_{2}}}}{|Q|} \int_{Q} \int_{Q^{*}}\left|f_{1}\left(y_{1}\right)\right| \int_{\left(Q^{*}\right)^{c}} \frac{\left|f_{2}\left(y_{2}\right)\right|}{\left|z-y_{2}\right|^{2 n}} \\
& \times\left(\omega\left(\frac{\left|z-x_{Q}\right|}{\left|z-y_{2}\right|}\right)+\frac{\left|z-x_{Q}\right|}{\left|z-y_{1}\right|+\left|z-y_{2}\right|}\right) d y_{2} d y_{1} d z \\
& \lesssim \frac{\left\|b_{1}\right\|{\dot{\Lambda_{\beta}}}_{\beta_{1}}\left\|b_{2}\right\|_{\dot{\wedge}_{\beta_{2}}}}{|Q|} \int_{Q} \int_{Q^{*}}\left|f_{1}\left(y_{1}\right)\right| \sum_{k=1}^{\infty} \int_{2^{k+3} \sqrt{n} Q \backslash 2^{k+2} \sqrt{n} Q} \frac{\left(\omega\left(2^{-k}\right)+2^{-k}\right)\left|f_{2}\left(y_{2}\right)\right|}{\left|2^{k} \sqrt{n} Q\right|^{2}} d y_{2} d y_{1} d z \\
& \lesssim\left\|b_{1}\right\|_{{\dot{\lambda_{\beta}}}_{\beta_{1}}}\left\|b_{2}\right\|_{{\dot{\aleph}}_{\beta_{2}} \frac{1}{|Q|}} \int_{Q^{*}}\left|f_{1}\left(y_{1}\right)\right| d y_{1} \\
& \times \sum_{k=1}^{\infty} \frac{|Q|}{\left|2^{k+3} \sqrt{n} Q\right|}\left(\omega\left(2^{-k}\right)+2^{-k}\right) \frac{1}{\left|2^{k+3} \sqrt{n} Q\right|} \int_{2^{k+3} \sqrt{n} Q \backslash 2^{k+2} \sqrt{n} Q}\left|f_{2}\left(y_{2}\right)\right| d y_{2} \\
& \lesssim\left\|b_{1}\right\|_{\dot{\wedge}_{\beta_{1}}}\left\|b_{2}\right\|_{{\dot{\lambda_{\beta}}}_{\beta_{2}}} M\left(f_{1}\right)(x) M\left(f_{2}\right)(x)
\end{aligned}
$$

Similarly, $\mathfrak{N}_{24} \lesssim\left\|b_{1}\right\|_{{\dot{\lambda}}_{1}}\left\|b_{2}\right\|_{{\dot{\lambda}}_{\beta_{2}}} M\left(f_{1}\right)(x) M\left(f_{2}\right)(x)$.

For $y_{1}, y_{2} \in\left(Q^{*}\right)^{c}$, we have $\left|y_{1}-x_{Q}\right| \sim\left|y_{1}-z\right|,\left|y_{2}-x_{Q}\right| \sim\left|y_{2}-z\right|$. Then by 
Minkowski's inequality and by Lemma 2.1.

$$
\begin{aligned}
& \mathfrak{N}_{25} \\
& \lesssim \frac{\left\|b_{2}\right\|_{\dot{\lambda}_{\beta_{2}}}}{|Q|^{1+\beta_{1} / n}} \int_{Q} \int_{\left(\mathbb{R}^{n}\right)^{2}}\left|\left(b_{1}\left(y_{1}\right)-\left(b_{1}\right)_{Q^{*}}\right)\right| \\
& \times \sup _{\eta}\left(\int_{0}^{\infty}\left|K_{t, \mu, \eta}\left(z, y_{1}, y_{2}\right)-K_{t, \mu, \eta}\left(x_{Q}, y_{1}, y_{2}\right)\right|^{2} \frac{d t}{t}\right)^{1 / 2}\left|f_{1}^{\infty}\left(y_{1}\right) f_{2}^{\infty}\left(y_{2}\right)\right| d y_{1} d y_{2} d z \\
& \lesssim \frac{\left\|b_{1}\right\|{\dot{\dot{\lambda}_{\beta_{1}}}}_{\left\|b_{2}\right\|_{\dot{\wedge}_{\beta_{2}}}}}{|Q|^{1+\beta_{1} / n}} \int_{Q} \int_{\left(\mathbb{R}^{n}\right)^{2}} \frac{\left|y_{1}-x_{Q}\right|^{\beta_{1}}\left|f_{1}^{0}\left(y_{1}\right) f_{2}^{\infty}\left(y_{2}\right)\right|}{\left(\left|z-y_{1}\right|+\left|z-y_{2}\right|\right)^{2 n}} \\
& \times\left(\omega\left(\frac{\left|z-x_{Q}\right|}{\left|z-y_{1}\right|+\left|z-y_{2}\right|}\right)+\frac{\left|z-x_{Q}\right|}{\left|z-y_{1}\right|+\left|z-y_{2}\right|}\right) d y_{1} d y_{2} d z \\
& \lesssim \frac{\left\|b_{1}\right\|{\dot{\lambda_{\beta}}}_{\beta_{1}}\left\|b_{2}\right\|{\dot{\lambda_{\beta}}}_{\beta_{2}}}{|Q|^{1+\beta_{1} / n}} \int_{Q} \int_{\left(\left(Q^{*}\right)^{c}\right)^{2}} \frac{\left|f_{1}\left(y_{1}\right)\right|\left|f_{2}\left(y_{2}\right)\right|}{\left|y_{1}-x_{Q}\right|^{2 n-\beta_{1}}} \\
& \times\left(\omega\left(\frac{\left|z-x_{Q}\right|}{\left|z-y_{1}\right|}\right)+\frac{\left|z-x_{Q}\right|}{\left|z-y_{1}\right|+\left|z-y_{2}\right|}\right) d y_{1} d y_{2} d z \\
& \lesssim \frac{\left\|b_{1}\right\|{\dot{\lambda_{\beta_{1}}}}\left\|b_{2}\right\|_{\dot{\lambda}_{\beta_{2}}}}{|Q|^{1+\beta_{1} / n}} \int_{Q} \sum_{k=1}^{\infty} \int_{2^{k+3} \sqrt{n} Q \backslash 2^{k+2} \sqrt{n} Q} \frac{\left|f_{1}\left(y_{1}\right)\right|\left|f_{2}\left(y_{2}\right)\right|}{\left|y_{1}-x_{Q}\right|^{2 n-\beta_{1}}} \\
& \times\left(\omega\left(\frac{\left|z-x_{Q}\right|}{\left|z-y_{1}\right|}\right)+\frac{\left|z-x_{Q}\right|}{\left|z-y_{1}\right|+\left|z-y_{2}\right|}\right) d y_{1} d y_{2} d z \\
& \lesssim\left\|b_{1}\right\|_{\dot{\wedge}_{\beta_{1}}}\left\|b_{2}\right\|_{\dot{\wedge}_{\beta_{2}}} \sum_{k=1}^{\infty} \frac{2^{k \beta_{1}}\left(\omega\left(2^{-k}\right)+2^{-k}\right)}{\left|2^{k+3} \sqrt{n} Q\right|^{2}} \int_{2^{k+3} \sqrt{n} Q \backslash 2^{k+2} \sqrt{n} Q}\left|f_{1}\left(y_{1}\right)\right| d y_{1} \\
& \times \int_{2^{k+3} \sqrt{n} Q \backslash 2^{k+2} \sqrt{n} Q}\left|f_{2}\left(y_{2}\right)\right| d y_{2} \\
& \lesssim\left\|b_{1}\right\|_{{\dot{\lambda}}_{1}}\left\|b_{2}\right\|_{{\dot{\lambda_{\beta}}}_{\beta_{2}}} M\left(f_{1}\right)(x) M\left(f_{2}\right)(x),
\end{aligned}
$$

where the assumption (1.4) was used. Combining the estimates for $\mathfrak{N}_{21}, \mathfrak{N}_{22}, \mathfrak{N}_{23}, \mathfrak{N}_{24}$, $\mathfrak{N}_{25}$, we get

$$
\mathfrak{N}_{2} \lesssim\left\|b_{1}\right\|_{\dot{\lambda}_{\beta_{1}}}\left\|b_{2}\right\|_{{\dot{\lambda_{\beta}}}_{\beta_{2}}}\left\{M_{r}\left(U^{*}\left(f_{1}, f_{2}\right)\right)(x)+M_{q_{1}}\left(f_{1}\right)(x) M_{q_{2}}\left(f_{2}\right)(x)+M\left(f_{1}\right)(x) M\left(f_{2}\right)(x)\right\}
$$

Finally, by Hölder's inequality, Lemma 2.2 and the estimate of Hardy-Littlewood maximal function, we obtain that

$$
\begin{aligned}
\left\|U_{\Pi \vec{b}}^{*}(\vec{f})\right\|_{\dot{F}_{p}^{\beta, \infty}} & \approx\left\|\sup _{Q} \frac{1}{|Q|^{1+\beta / n}} \int_{Q}\left|U_{\Pi \vec{b}}^{*}(\vec{f})(z)-\left(U_{\Pi \vec{b}}^{*}(\vec{f})\right)_{Q}\right| d z\right\|_{L^{p}} \\
& \lesssim\left\|b_{1}\right\|_{{\dot{\lambda_{\beta}}}_{\beta_{1}}}\left\|b_{2}\right\|_{{\dot{\lambda_{\beta}}}_{\beta_{2}}}\left\|f_{1}\right\|_{L^{p_{1}}}\left\|f_{2}\right\|_{L^{p_{2}}} .
\end{aligned}
$$

This finishes the proof of Theorem 1.3 . 


\section{Acknowledgments}

The first author was supported partly by the Key Research Project for Higher Education in Henan Province (No. 19A110017). The second author was supported partly by NSFC (Nos. 11671039, 11871101) and NSFC-DFG (No. 11761131002). The third author was supported partly by NSFC (No. 11571160).

\section{References}

[1] B. T. Anh and X. T. Duong, On commutators of vector BMO functions and multilinear singular integrals with non-smooth kernels, J. Math. Anal. Appl. 371 (2010), no. $1,80-84$.

[2] X. Chen, Q. Xue and K. Yabuta, On multilinear Littlewood-Paley operators, Nonlinear Anal. 115 (2015), 25-40.

[3] R. R. Coifman, D. G. Deng and Y. Meyer, Domaine de la racine carrée de certains opérateurs différentiels accrétifs, Ann. Inst. Fourier (Grenoble) 33 (1983), no. 2, 123134.

[4] R. R. Coifman and Y. Meyer, Au delà des opérateurs pseudo-différentiels, Astérisque 57, Société Mathématique de France, Paris, 1978.

[5] G. David and J.-L. Journé, Une caractérisation des opérateurs intégraux singuliers bornés sur $L^{2}\left(\mathbf{R}^{n}\right)$, C. R. Acad. Sci. Paris Sér. I Math. 296 (1983), no. 18, 761-764.

[6] E. B. Fabes, D. S. Jerison and C. E. Kenig, Multilinear Littlewood-Paley estimates with applications to partial differential equations, Proc. Nat. Acad. Sci. U.S.A. 79 (1982), no. 18, 5746-5750.

[7] _ Multilinear square functions and partial differential equations, Amer. J. Math. 107 (1985), no. 6, 1325-1368.

[8] L. Grafakos, D. He and P. Honzík, Rough bilinear singular integrals, Adv. Math. 326 (2018), 54-78.

[9] L. Grafakos, L. Liu and D. Yang, Multiple-weighted norm inequalities for maximal multi-linear singular integrals with non-smooth kernels, Proc. Roy. Soc. Edinburgh Sect. A 141 (2011), no. 4, 755-775.

[10] L. Grafakos and Z. Si, The Hörmander multiplier theorem for multilinear operators, J. Reine Angew. Math. 668 (2012), 133-147. 
[11] J. Hart, Bilinear square functions and vector-valued Calderón-Zygmund operators, J. Fourier Anal. Appl. 18 (2012), no. 6, 1291-1313.

[12] M. Hormozi, Z. Si and Q. Xue, On general multilinear square function with nonsmooth kernels, Bull. Sci. Math. 149 (2018), 1-22.

[13] G. Lu and P. Zhang, Multilinear Calderón-Zygmund operators with kernels of Dini's type and applications, Nonlinear Anal. 107 (2014), 92-117.

[14] D. Maldonado and V. Naibo, Weighted norm inequalities for paraproducts and bilinear pseudodifferential operators with mild regularity, J. Fourier Anal. Appl. 15 (2009), no. 2, 218-261.

[15] H.-x. Mo and S.-z. Lu, Commutators generated by multilinear Calderón-Zygmund type singular integral and Lipschitz functions, Acta Math. Appl. Sin. Engl. Ser. 30 (2014), no. 4, 903-912.

[16] M. Paluszyński, Characterization of the Besov spaces via the commutator operator of Coifman, Rochberg and Weiss, Indiana Univ. Math. J. 44 (1995), no. 1, 1-17.

[17] S. Sato and K. Yabuta, Multilinearized Littlewood-Paley operators, Sci. Math. Jpn. 55 (2002), no. 3, 447-453.

[18] S. Shi, Q. Xue and K. Yabuta, On the boundedness of multilinear Littlewood-Paley $g_{\lambda}^{*}$ function, J. Math. Pures Appl. (9) 101 (2014), no. 3, 394-413.

[19] Z. Si and Q. Xue, Multilinear square functions with kernels of Dini's type, J. Funct. Spaces 2016 (2016), Art. ID 4876146, 11 pp.

[20] _ Estimates for iterated commutators of multilinear square fucntions with Dinitype kernels, J. Inequal. Appl. 2018 (2018), Paper No. 188, 21 pp.

[21] Z. Si and P. Zhang, Iterated commutators of multilinear Calderón-Zygmund maximal operators on some function spaces, J. Inequal. Appl. 2019 (2019), Paper No. 100, 21 pp.

[22] J. Sun and P. Zhang, Commutators of multilinear Calderón-Zygmund operators with Dini type kernels on some function spaces, J. Nonlinear Sci. Appl. 10 (2017), no. 9, 5002-5019.

[23] N. Tomita, A Hörmander type multiplier theorem for multilinear operators, J. Funct. Anal. 259 (2010), no. 8, 2028-2044. 
[24] W. Wang and J.-s. Xu, Commutators of multilinear singular integrals with Lipschitz functions, Commun. Math. Res. 25 (2009), no. 4, 318-328.

[25] Q. Xue, X. Peng and K. Yabuta, On the theory of multilinear Littlewood-Paley gfunction, J. Math. Soc. Japan 67 (2015), no. 2, 535-559.

[26] Q. Xue and J. Yan, On multilinear square function and its applications to multilinear Littlewood-Paley operators with non-convolution type kernels, J. Math. Anal. Appl. 422 (2015), no. 2, 1342-1362.

[27] K. Yabuta, A multilinearization of Littlewood-Paley's g-function and Carleson measures, Tohoku Math. J. (2) 34 (1982), no. 2, 251-275.

\section{Zengyan Si}

School of Mathematics and Information Science, Henan Polytechnic University, Jiaozuo 454000, China

E-mail address: zengyan@hpu.edu.cn

Qingying Xue

School of Mathematical Sciences, Beijing Normal University, Laboratory of Mathematics and Complex Systems, Ministry of Education, Beijing 100875, China

E-mail address: qyxue@bnu.edu.cn

Pu Zhang

Department of Mathematics, Mudanjiang Normal University, Mudanjiang, 157011, China E-mail address: puzhang@sohu.com 